\title{
Tandem Mobility Mass Spectrometry Study of Electrosprayed Tetraheptyl Ammonium Bromide Clusters
}

\author{
Juan Fernandez de la Mora \\ Department of Mechanical Engineering, Yale University, New Haven, Connecticut, USA
}

Bruce A. Thomson

MDS Sciex, Concord, Ontario, Canada

Manuel Gamero-Castaño
Jet Propulsion Laboratory, Pasadena, California, USA

Multiply charged electrospray ions from concentrated solutions of Heptyl $\mathrm{N}^{+} \mathrm{Br}^{-}$(designated $\mathrm{A}^{+} \mathrm{B}^{-}$hereafter) in formamide are analyzed mass spectrometrically (MS) following mobility selection in ambient air in a differential mobility analyzer (DMA). Most of the sharp mobility peaks seen are identified as $(A B)_{n} A^{+}$clusters, with $0 \leq n \leq 5$. One anomalously abundant and mobile ion is identified as $\mathrm{NH}_{4}^{+}(\mathrm{AB})_{4}$. Six ions in the $(\mathrm{AB})_{n}\left(\mathrm{~A}^{+}\right)_{2}$ series are also identified, completing and correcting earlier mobility data for singly and doubly charged ions up to masses of almost $9000 \mathrm{Da}$. The more mobile of two broad humps seen in the mobility spectrum includes $\mathrm{m} / \mathrm{z}$ values approximately from 2500 up to $12,000 \mathrm{Da}$. It is formed primarily by multiply charged $(\mathrm{AB})_{\mathrm{n}}\left(\mathrm{A}^{+}\right)_{\mathrm{z}}$ clusters with multiple ammonium bromide adducts. Because of overlapping of many peaks of different $\mathrm{m} / \mathrm{z}$ and charge state $\mathrm{z}$, only a few individual species can be identified by MS alone in this highly congested region. However, the spectral simplification brought about by mobility selection upstream of the MS reveals a series of broad modulations in $\mathrm{m} / \mathrm{z}$ space, with all ions resolved in the second, third, ... sixth modulation being in charge states $z=2,3, \ldots 6$, respectively. Extrapolation of this trend beyond the sixth wave fixes the ion charge state (in some cases up to $\mathrm{z}=15$ ) and mass (beyond $\mathrm{m}=175,000 \mathrm{u}$ ). This wavy structure had been previously observed and explained in terms of ion evaporation kinetics from volatile drops, though without mass identification. All observations indicate that the clusters are formed as charged residues, but their charge state is fixed by the IribarneThomson ion evaporation mechanism. Consequently, the measured curve of cluster diameter versus $\mathrm{z}$ yields the two parameters governing ion evaporation kinetics. Clusters with $\mathrm{z}>1$ and electrical mobility $\mathrm{Z}>0.495 \mathrm{~cm}^{2} / \mathrm{V} / \mathrm{s}$ are metastable and evaporate a singly charged cluster, probably $(\mathrm{AB})_{2} \mathrm{~A}^{+}$, between the DMA and the MS. Plotting the electrical mobilities $\mathrm{Z}$ of the clusters in the form $(\mathrm{z} / \mathrm{Z})^{1 / 2}$ versus $\mathrm{m}^{1 / 3}$ (both proportional to cluster diameter) collapse the data for all cluster sizes and charge states into one single straight line for Z below 0.495 $\mathrm{cm}^{2} / \mathrm{V} / \mathrm{s}$. This linear relation reveals a uniform apparent cluster density of $0.935 \mathrm{~g} / \mathrm{cm}^{3}$ and an effective hard-sphere diameter of the air molecules of $0.44 \mathrm{~nm}$. An anomalous mobility increase is observed at diameters below $3 \mathrm{~nm}$. (J Am Soc Mass Spectrom 2005, 16, 717-732) (C) 2005 American Society for Mass Spectrometry

$\mathrm{F}$ ollowing the pioneering study of Meng and Fenn [1], numerous mass spectrometric (MS) studies have examined the multiply charged aggregates forming upon electrospraying (ES) solutions of ionic and nonionic solutes. Such investigations have been motivated by reasons as varied as identifying the source of the chemical noise often present in mass spectra [2],

Published online March 11, 2005

Address reprint requests to Dr. J. Fernandez de la Mora, Department of Mechanical Engineering, Yale University, P.O. Box 208286, New Haven, CT 06520-8286, USA. E-mail: juan.delamora@yale.edu or establishing the origin of relatively large and multiply charged electrospray ions [3-10]. Most ES-MS studies with solutions $\mathrm{M}^{+} \mathrm{X}^{-}$of salts of monovalent ions electrosprayed in the positive mode have revealed the dominant presence of the cation $\mathrm{M}^{+}$in various states of solvation, as well as a weaker series of singly charged clusters of the form $(\mathrm{MX})_{\mathrm{n}} \mathrm{M}^{+}$. In some exceptional instances primarily involving relatively large cations or anions (such as $\mathrm{ClO}_{4}^{-}$and $\mathrm{Cs}^{+}$), multiply charged clusters of the form $(\mathrm{MX})_{\mathrm{n}} \mathrm{M}^{+\mathrm{z}}$ have also been observed, though few such studies have reported charge states beyond $z=2$. Considerably higher charge states have 
been found from electrosprays of small peptides such as arginine (up to $z=4$ in [1]), and their stability has been rationalized as due to relatively strong hydrogen bonding between the $\mathrm{n}$ monomer molecules composing the cluster [11]. A recent mobility-MS investigation on multiply charged clusters [2] reports $(\mathrm{n}, \mathrm{z})$ values as large as $(75,7)$ for tetrapeptides.

An alternative means used to unscramble the complex distributions of cluster ions resulting from the two (at least) independent degrees of freedom $\mathrm{n}$ and $\mathrm{z}$ has recently been used to provide strong support for the ion evaporation model of Iribarne and Thomson [12]. It has involved mobility selection in a first differential mobility analyzer (DMA, a device that separates ions in space according to their electrical mobility, and yields at its outlet only ions having a preselected mobility Z) followed by charge reduction and a second mobility determination [13]. The clusters are formed as charged residues after complete drop evaporation. By assuming that the cluster charge $\mathrm{z}$ is equal to that on a solvent drop of the same diameter $d$, the observed $z(d)$ relation was seen to be consistent with the Iribarne-Thomson ion evaporation mechanism, yielding the two parameters governing its kinetics. The same authors have used the simpler approach of first reducing the cluster charge to unity, and then measuring cluster mobility $[14,15]$. In principle, this second scheme loses information on the original charge state, which is of primary importance in studies on the mechanism of electrospray ionization. However, it turns out that the mobility distribution of these singly charged clusters exhibits a remarkable series of modulations in the amplitude of the various peaks [15]. The first, second, third, etc. modulations were shown via the tandem DMA method to correspond to clusters originally charged with one, two, three, etc. charges $[13,15]$. Hence, information of the original cluster charge is not lost upon charge reduction, being instead preserved in the modulated amplitude distribution just described. This charge-reduction technique, therefore, simplifies the determination of the relation between cluster mobility (hence, after suitable calibration, size, and mass) and charge, from which to infer ion evaporation kinetics.

In spite of the valuable information provided by pure mobility analysis, combining it with mass spectrometry would help clarify a number of important remaining issues. First, although the mobility of spheres in the free-molecule range has been studied widely, the established relation between mobility and radius is not reliable in the nanometer range. Hence, the particle diameter (of paramount relevance to the kinetics of ion evaporation) remains ambiguously related to the measured cluster mobility. Second, these mobility studies show cluster abundances quite comparable for all charge states in the range of $\mathrm{z}$ between at least 1 and 9 . In contrast, mass spectrometric studies show overwhelmingly dominant peaks for singly charged clusters, and, as already discussed, rarely see any cluster with $z>1$. These discrepancies may be due to many reasons, such as differences in the solutions (salt concentration and solvent composition) and spraying parameters; the relatively large losses of ions in the sampling tubes of the DMA (which would pass preferentially the larger and less mobile clusters); the limited stability of most multiply charged clusters, which might then be readily observable in the DMA at atmospheric pressure, but could conceivably disintegrate in their passage to the low pressure region of the mass spectrometer etc. In view of these circumstances, the objective of the present work is to analyze mass spectrometrically the same solutions, sprayed with the same electrospray source, after passing the clusters through a DMA closely related to that used in references [13-15]. The broad conclusions to be reached are similar to those of $[14,15]$, but the much greater level of information afforded by mass spectrometry will shed new light into the problem.

The plan of this study is as follows. After describing the experimental system and methods, we infer cluster mobilities, charge states and masses from DMA-MS measurements. We then establish the relation between mobility, mass, and size. This then permits comparing measured cluster diameter versus charge curves with predictions from the ion evaporation model, which yields the corresponding activation energy and curvature correction parameter. Next we examine whether there is reasonable ground to doubt that ions do evaporate from charged evaporating drops, and answer it negatively. Our main findings are then summarized.

Before proceeding, a clarification is due on our unconventional salt and solvent, and for our highly concentrated solutions. Formamide's main advantage is its low volatility. This facilitates its spraying at very low flow rates from capillary bores as wide as $40 \mu \mathrm{m}$ or more, without a risk that solvent evaporation from the Taylor cone will lead to solute precipitation, even from concentrated solutions. A large cation (or anion) offers the advantage that the spectral complexity at a given mass is much smaller than with a smaller salt. A large concentration of an involatile salt is essential when the goal pursued is to form clusters covering a wide range of sizes and charges in order to determine experimentally how these two key variables are related.

\section{Experimental}

Tetraheptyl ammonium bromide and formamide were obtained from Sigma (St. Louis, MO). 2-Propanol was from Baker (Phillipsburg, NJ). All were used as received. Note that formamide is a dangerous teratogen.

A schematic of the experimental setup is shown in Figure 1. The sample solution is introduced in a polypropylene vial $\left(1 \mathrm{~cm}^{3}\right.$ capacity) and put in electrical contact with a high voltage power supply through a platinum electrode. One end of a silica tube ( $40 \mu \mathrm{m}$ i.d., $360 \mu \mathrm{m}$ o.d.; Polymicro Technologies, Phoenix, AZ) is immersed in the liquid sample, while its other end is sharpened into a conical tip and goes inside a small 


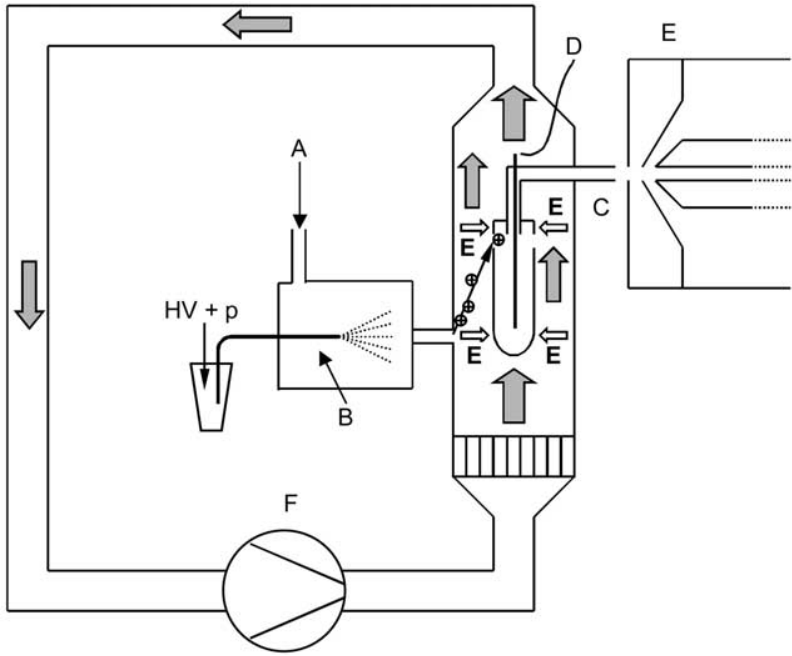

Figure 1. Sketch of the experimental arrangement. A, Inlet for dry air to carry ES ions into the DMA. B, Electrospray needle. C, ion outlet from DMA leading to the vicinity of the inlet pinhole of the mass spectrometer. D, DMA collecting electrode connected to an electrometer. E: TOF 365 mass spectrometer. F: Pump driving the recirculating sheath flow. The DMA is located on the right branch of the recirculating loop. The gas is laminarized at its bottom, flows around a bullet-shaped central electrode into the annular region between two cylinders, where ions are injected through a slit in the outer cylinder. Ions are separated in space by the electric field $\mathbf{E}$ according to their mobilities, and only those with a preselected $Z$ value are sampled through a slit on the inner electrode and sent to the mass spectrometer.

stainless steel cross acting as the electrospray chamber. The spraying tip is illuminated and observed through two of the arms of the cross, provided with windows. The spray is sampled through a short tube $(1 / 4$ in. o.d., $4 \mathrm{~mm}$ i.d.) inserted in the fourth arm of the cross, and brought into the inlet port of the differential mobility analyzer (DMA). This analyzer is sketched in the center of Figure 1. It combines an axial high-speed stream of dry air (the sheath gas) with a radial electric field in the annular region between two concentric cylinders. A flow of ion-laden gas is injected in the analyzing region through a circular slit in the outer cylinder, at a flow rate typically much smaller than the sheath gas (1-10 $1 /$ min versus some $5001 / \mathrm{min}$ ). Ions then follow mobility-dependent trajectories, carried axially by the gas and radially by the field. At fixed sheath gas flow rate and voltage difference between the two cylindrical electrodes, only the ions whose electrical mobility $\mathrm{Z}$ is close to a preset value reach a second sampling slit in the inner electrode and are drawn into the ion outlet port of the DMA. The sheath gas flow was recirculated into the DMA through a vacuum cleaner pump (max power $=$ $1.4 \mathrm{~kW}$ ), ensuring that the same flow rate of ions forced through the electrospray chamber (regulated upstream through a rotameter) into the DMA would exit through the DMA ion outlet. The mobility-selected ion-laden flow was then blown into the vicinity of the atmospheric pressure inlet hole of the mass spectrometer. The mass spectrometer samples only a small fraction of these $10 \mathrm{l} / \mathrm{min}$, but the large flow rates through the DMA are meant to reduce the already very large ion losses through it, and hence increase the ion density in the sampling region of the MS. The vast majority of the ions ingested at the entrance of the sampling tube facing the ES needle are in fact lost by space charge repulsion in the tube. Typically, a few tens of $\mathrm{nA}$ are received by the tube, and only some $100 \mathrm{pA}$ reach the exit of the DMA. This inefficient transmission will be compensated in part by the relatively high transmission efficiency of the mass spectrometer, and by use of unusually long acquisition periods in the mass spectrometer. The electrical mobility is strictly proportional to the ratio of gas flow rate over voltage difference, with a proportionality constant known exactly for a cylindrical geometry [16]. However, because the gas flow rate cannot be measured with high accuracy, we determine $\mathrm{Z}$ by using the mobility of the dimer ion $\mathrm{A}^{+}(\mathrm{AB})_{1}(\mathrm{~A}=$ tetraheptyl ammonium, $\mathrm{B}=$ bromide) as a reference. The mobility of this ion in air $\left(1 / \mathrm{Z}=1.51 \mathrm{Vs} \mathrm{cm}^{-2}\right.$ at standard conditions) has been previously obtained in [14] via careful measurement of the gas flow rate. The operating temperature was very close to room temperature. The electrospray chamber was raised to the same voltage as the outer DMA electrode, which varied from 0 up to several $\mathrm{kV}$. This mode of operation is very dangerous and should never be used without special precautions. The electrospray current is monitored in an ammeter located between the high voltage supply and the spraying needle. Its stability was high, to the point that no discernible changes in the current were seen even in overnight experiments lasting 8 or $9 \mathrm{~h}$. In some initial experiments the DMA voltage (fixing the selected mobility) was slowly ramped while mass spectra were continuously acquired. This two-dimensional scan was suitable to investigate the most abundant clusters, which were either singly or doubly charged. For all the data obtained with multiply charged clusters, the weakness of the signal required fixing the DMA voltage and accumulating mass spectral data for long periods ranging from $10 \mathrm{~min}$ to several hours.

The mass spectrometer was a quadrupole time-offlight (QqTOF) tandem mass spectrometer system [17], used for these experiments almost exclusively in the single MS mode. All mass analysis was performed with the TOF section, which has a mass resolution of about 10,000 and mass accuracy of $5 \mathrm{ppm}$ with internal calibration. Ions were sampled from the outlet of the DMA through a $0.25 \mathrm{~mm}$ orifice into the vacuum system. Control of electric fields in the region of gas expansion into the vacuum can be used to fragment ions further; however, in all of the experiments reported here, these fields were operated so as to reduce or eliminate fragmentation in order to retain the identity of the original mobility-separated species. The presence of clean mobility-resolved peaks of singly charged clusters up to the 5-mer, without the presence of fragments in the mass spectrum, shows that this appeared to be successful. In addition, there was no evidence in 
any of the spectra of clustering with water vapor in the free jet region. All data acquisition was performed with the TOFMA program, courtesy of Werner Ens, University of Manitoba.

\section{Results and Discussion}

\section{Singly Charged Clusters}

Figure $2 \mathrm{a}$ is a mobility spectrum for a solution of $20 \mathrm{mM}$ tetraheptyl ammonium bromide in ethanol. It superposes the total ion current signal from the mass spectrometer and the current collected with an electrometer at the DMA exit (the flat-top signal corresponding to the monomer and dimer peaks is attributable to saturation of the current amplifier). The electrometer data were recorded as a function of DMA voltage $V$, which was turned into a mobility based on the known value of the dimer peak. The accuracy of this procedure was verified by comparing the resulting mobilities of the five most prominent peaks with the values reported in [14]. The agreement was within $1 \%$ for all except the second and first peaks ( $2 \%$ difference).

The MS data were taken in a chromatographic mode, where the voltage was stepped linearly with time and mass spectra were recorded continuously at a rate of approximately one spectrum per second. The relation between time and voltage was known, and the voltage was again turned into a mobility based on the known value of the dimer peak. Comparison with the data of
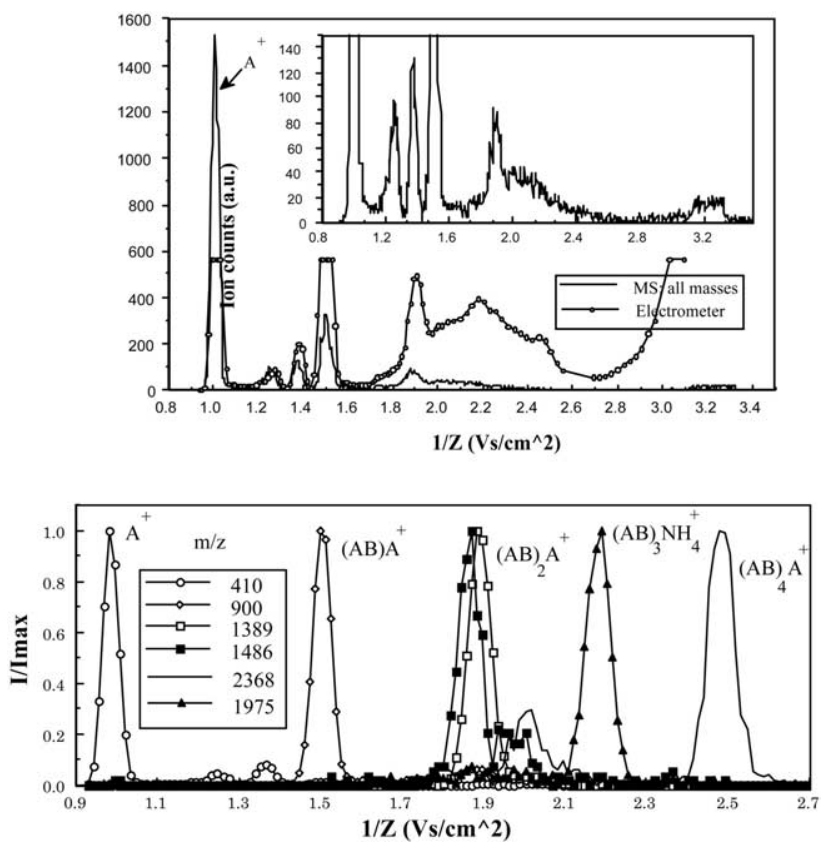

Figure 2. Mobility spectra of electrospray ions from tetraheptyl ammonium bromide. All mass channels are shown at top, either as measured in the MS detector, or with an electrometer upstream of the MS pinhole. Mass-selected spectra for singly charged clusters $\mathrm{A}^{+}(\mathrm{AB})_{\mathrm{n}}$ and $\mathrm{A}^{+}(\mathrm{AB})_{\mathrm{n}} \mathrm{NH}_{4} \mathrm{Br}$ are shown at bottom. The secondary peaks between $\mathrm{A}^{+}$and $(\mathrm{AB}) \mathrm{A}^{+}$correspond to doubly charged clusters having fragmented between the DMA and the MS.
[14] gives excellent agreement (better than 1\%) for all the peaks, except the monomer ion, for which we obtain $1 / \mathrm{Z}=0.992 \mathrm{Vs} / \mathrm{cm}^{2}$. The difference with [14] (1.04 $\mathrm{Vs} / \mathrm{cm}^{2}$ ) is seen both in the MS-selected data of Figure $2 \mathrm{~b}$ as well as in those of Figure 2a. It may be attributable to the fact that the solvent here is ethanol, and that the formamide solvent used in [14] may tend to solvate the monomer. The two spectra shown in Figure 2a are rather similar, in spite of the fact that the first represents the signal detected using ion counting at the TOF detector, while the latter was recorded with a current meter (electrometer) before ions entered the transfer tube to the MS. The response of the electrometer is proportional to the charge of the ions, while the response of the TOF detector (a multi-channel plate operated with individual ion counting) is generally not. High $m / z$ ions are detected less efficiently by the TOF (sensitivity starting to decrease beyond $\mathrm{m} / \mathrm{z} 2000$ ), but higher $\mathrm{m} / \mathrm{z}$ ions are probably more efficiently transmitted from the DMA to the MS. The various discrimination factors may thus largely cancel out. However, the most obvious difference is that the large low-mobility peak in the electrometer mobility spectrum is almost absent in the MS-recorded spectrum. Examination of the mass-resolved mobility spectra shows that essentially all the ions seen at the inset of Figure 2a in the vicinity of $3.2 \mathrm{Vs} / \mathrm{cm}^{2}$ are associated to the monomer, dimer, and trimer ions $(A B)_{n} A^{+}(n=0,1,2)$, surely emitted from larger particles or drops after DMA selection. This suggests that the particles classified by the DMA at about $3.2 \mathrm{Vs} / \mathrm{cm}^{2}$ are either beyond the $\mathrm{m} / \mathrm{z}$ range of the TOF $(40,000 \mathrm{u})$, or else so large as to be virtually undetectable at the TOF detector. These particles have been observed and interpreted in earlier tandem DMA experiments as due to large salt residues from the evaporation of electrosprayed mother drops, which never reached the ion evaporation regime [14].

Several normalized mass-selected mobility spectra are shown in Figure $2 b$ for the most prominent ions. Some mass peaks exhibit multiple mobility peaks. For instance, the tetraheptyl ammonium cation $\mathrm{A}^{+}$shows a primary peak at $1 / \mathrm{Z}=0.99 \mathrm{Vs} / \mathrm{cm}^{2}$, and two smaller peaks, $\mathrm{A}_{1}^{+}$and $\mathrm{A}_{2}^{+}$, at 1.25 and $1.37 \mathrm{Vs} / \mathrm{cm}^{2}$, which are either isomeric forms of $\mathrm{A}^{+}$(possible but unlikely), or doubly charged ions which fragment after going through the DMA. We had originally rejected the second hypothesis because $\mathrm{A}^{+}$is the only MS ion associated to $1 / \mathrm{Z}$ values of 1.25 and $1.37 \mathrm{Vs} / \mathrm{cm}^{2}$, while a large doubly charged ion would ordinarily fragment into two different ions. However, we later noticed that an ion of that mobility was unambiguously identified in reference [14] as doubly charged, suggesting that these doubly charged ions fragment symmetrically following the DMA, forming a pair of $\mathrm{A}^{+}$ions and neutral species. The ion labeled $(\mathrm{AB})_{4} \mathrm{~A}^{+}(m / z=1975)$ also shows two mobility peaks, one of them associated to a doubly charged ion. Table 1, reports masses, abundances (all mobility channels), structures, and mobilities for the main peaks observed. Note two additional ions incor- 
Table 1. Composition, mass (first isotope), mobility, and relative abundance of the dominant singly charged ions observed

\begin{tabular}{|c|c|c|c|c|c|}
\hline Ion & Mass (u) & $1 / \mathrm{Z}\left(\mathrm{Vs} / \mathrm{cm}^{2}\right)$ & Abundance $^{a}$ & $1 / Z$ in $[14]$ & $\begin{array}{c}\text { Ion assignment } \\
\text { in [14] }\end{array}$ \\
\hline $\mathrm{A}^{+}$ & 410.47 & 0.992 & 23000 & 1.04 & $\mathrm{~A}^{+}$ \\
\hline$(\mathrm{AB}) \mathrm{A}^{+}$ & 899.86 & 1.51 & 8000 & 1.508 & $(A B)_{2} A^{+}$ \\
\hline$(\mathrm{AB})_{2} \mathrm{~A}^{+}$ & 1389.25 & 1.897 & 2500 & 1.88 & $(A B)_{3} A^{+}$ \\
\hline$(\mathrm{AB})_{3} \mathrm{NH}_{4}^{+}$ & 1486.21 & 1.87 & 176 & & \\
\hline$(\mathrm{AB})_{3} \mathrm{~A}^{+}$ & 1878.64 & 2.26 & 130 & & \\
\hline$(\mathrm{AB})_{4} \mathrm{NH}_{4}{ }^{+}$ & 1975.60 & 2.19 & 415 & 2.19 & $(\mathrm{AB})_{4} \mathrm{~A}^{+}$ \\
\hline$(\mathrm{AB})_{4} \mathrm{~A}^{+}$ & 2368.04 & 2.49 & 418 & 2.46 & $(A B)_{5} A^{+}$ \\
\hline$(A B)_{5} A^{+}$ & 2857.43 & 2.83 & 142 & 2.83 & $(\mathrm{AB})_{6} \mathrm{~A}^{+}$ \\
\hline
\end{tabular}

aBased on the height of the ion peak including all mobility channels.

porating an extra $\mathrm{NH}_{4} \mathrm{Br}$ ion pair to the main series $(\mathrm{AB})_{\mathrm{n}} \mathrm{A}^{+}$. The adduct of $\mathrm{NH}_{4} \mathrm{Br}$ on $(\mathrm{AB})_{3} \mathrm{~A}^{+}$is likely composed of a central ammonium ion with its four hydrogen atoms tied to one salt molecule each: $\mathrm{NH}_{4}^{+}(\mathrm{AB})_{4}$. This cluster is far more abundant than its nonadducted counterpart $(\mathrm{AB})_{3} \mathrm{~A}^{+}$(Table 1), even though no ammonium was actively introduced in solution. We are much indebted to Professor Mark Johnson of Yale's Chemistry Department for pointing out that this singularity appears to indicate that much of the ammonium impurity present in solution has a strong tendency to bind to four ion pairs. Since all the singly charged ions observed have previously been shown to be formed by ion evaporation rather than as charged residues [14], this peculiarity of the solution phase would be similarly reflected on the electrosprayed ions, as observed.

Interestingly, the mobility of the larger ion $\mathrm{NH}_{4}^{+}(\mathrm{AB})_{3}$ is slightly larger that of the smaller ion $(\mathrm{AB})_{2} \mathrm{~A}^{+}$, revealing a more compact structure, probably also more symmetric (see [18] for the subtle connection between an extremum in the mobility and structural symmetry). The other adducted ion, $\mathrm{NH}_{4}^{+}(\mathrm{AB})_{4}$ exhibits also anomalously large mobility for its mass, further pointing to the more symmetric structure (tetrahedral in this case) hypothesized earlier.

\section{Doubly Charged Ions}

Weak but distinct peaks can be recognized in the mass spectrum at the $\mathrm{m} / \mathrm{z}$ values associated to doubly charged ions $(A B)_{n}\left(A^{+}\right)_{2}$ for $n=7,9$, and 11, The first two exhibit single mobility peaks, with corresponding values shown in Table 2 (note that we have shown the $\mathrm{m} / \mathrm{z}$ values corresponding to the average isotopic mass in 2 and 3, because we wish to consider the average mass of the particle in relation to the size and mobility in the following discussions). The mass peak associated to $\mathrm{n}=11$ exhibits several mobility peaks, of which only one is less mobile than $(\mathrm{AB})_{9}\left(\mathrm{~A}^{+}\right)_{2}$ and can therefore be associated to $(\mathrm{AB})_{11}\left(\mathrm{~A}^{+}\right)_{2}$. The mass peaks for the even values $\mathrm{n}=8,10$, and 12 show mobility distributions with dominant peaks associated with singly charged ions, and secondary peaks which can be attributed unambiguously to $(\mathrm{AB})_{8}\left(\mathrm{~A}^{+}\right)_{2}$ and $(\mathrm{AB})_{10}\left(\mathrm{~A}^{+}\right)_{2}$. The mobility spectrum for $(\mathrm{AB})_{12}\left(\mathrm{~A}^{+}\right)_{2}$ shows also a few isolated counts in the close vicinity of $1 / \mathrm{Z}=2.51$ $\mathrm{Vs} / \mathrm{cm}^{2}$ which has been included because it falls continuously in the series of the other five doubly charged peaks. The weak signals encountered here for doubly charged species contrast with those observed in [14]. The mobilities observed correspond almost exactly to those of [14], showing that the earlier mass assignment

Table 2. Composition, mass (average isotopic distribution) and mobility of the dominant doubly charged ions observed, complemented with the data of [14]

\begin{tabular}{|c|c|c|c|c|}
\hline Ion & $m / z(u)$ & $1 / \mathrm{Z}\left(\mathrm{Vs} / \mathrm{cm}^{2}\right)$ & $1 / Z$ in $[14]$ & $\begin{array}{c}\text { Ion assignment } \\
\text { in [14] }\end{array}$ \\
\hline$(A B)_{2}\left(A^{+}\right)_{2}$ & 901.48 & 1.255 & & $(\mathrm{AB})_{3}\left(\mathrm{~A}^{+}\right)_{2}$ \\
\hline$(A B)_{3}\left(A^{+}\right)_{2}$ & 1146.83 & 1.37 & 1.38 & $(\mathrm{AB})_{4}\left(\mathrm{~A}^{+}\right)_{2}$ \\
\hline$(A B)_{4}\left(A^{+}\right)_{2}$ & 1392.17 & & 1.53 & $(\mathrm{AB})_{5}\left(\mathrm{~A}^{+}\right)_{2}$ \\
\hline$(A B)_{5}\left(A^{+}\right)_{2}$ & 1637.52 & & 1.64 & $(A B)_{6}\left(A^{+}\right)_{2}$ \\
\hline$(A B)_{6}\left(A^{+}\right)_{2}$ & 1882.87 & & 1.80 & $(\mathrm{AB})_{7}\left(\mathrm{~A}^{+}\right)_{2}$ \\
\hline$(A B)_{7}\left(A^{+}\right)_{2}$ & 2128.21 & 1.88 & $1.87 \& 1.91$ & $(\mathrm{AB})_{8}\left(\mathrm{~A}^{+}\right)_{2}$ \\
\hline$(A B)_{8}\left(A^{+}\right)_{2}$ & 2373.56 & 2.02 & 2.01 & $(\mathrm{AB})_{9}\left(\mathrm{~A}^{+}\right)_{2}$ \\
\hline$(A B)_{9}\left(A^{+}\right)_{2}$ & 2618.91 & 2.143 & 2.13 & $(A B)_{10}\left(A^{+}\right)_{2}$ \\
\hline$(A B)_{10}\left(A^{+}\right)_{2}$ & 2864.25 & 2.278 & 2.26 & $(\mathrm{AB})_{11}\left(\mathrm{~A}^{+}\right)_{2}$ \\
\hline$(A B)_{11}\left(A^{+}\right)_{2}$ & 3109.6 & 2.42 & 2.40 & $(\mathrm{AB})_{12}\left(\mathrm{~A}^{+}\right)_{2}$ \\
\hline$(\mathrm{AB})_{12}\left(\mathrm{~A}^{+}\right)_{2}$ & 3354.95 & 2.52 & 2.50 & $(\mathrm{AB})_{13}\left(\mathrm{~A}^{+}\right)_{2}$ \\
\hline$(A B)_{13}\left(A^{+}\right)_{2}$ & 3600.29 & & 2.62 & $(\mathrm{AB})_{14}\left(\mathrm{~A}^{+}\right)_{2}$ \\
\hline$(\mathrm{AB})_{14}\left(\mathrm{~A}^{+}\right)_{2}$ & 3845.64 & & 2.73 & $(A B)_{15}\left(A^{+}\right)_{2}$ \\
\hline$(\mathrm{AB})_{15}\left(\mathrm{~A}^{+}\right)_{2}$ & 4090.99 & & 2.84 & $(\mathrm{AB})_{16}\left(\mathrm{~A}^{+}\right)_{2}$ \\
\hline$(\mathrm{AB})_{16}\left(\mathrm{~A}^{+}\right)_{2}$ & 4336.33 & & 2.92 & $(\mathrm{AB})_{17}\left(\mathrm{~A}^{+}\right)_{2}$ \\
\hline
\end{tabular}


was shifted up by one ion pair (AB). This shift could have been predicted just from the analogous shift previously identified in the mass assignment of [14] for the singly charged series. The second mobility peak at $\mathrm{Z}$ $=1.91 \mathrm{Vs} / \mathrm{cm}^{2}$, interpreted in [14] as a second isomer of what was taken to be $(\mathrm{AB})_{8}\left(\mathrm{~A}^{+}\right)_{2}$, is not seen here. Table 2 collects also the complete list of doubly charged ions compiled in [14], as well as the extra point associated to $(\mathrm{AB})_{2}\left(\mathrm{~A}^{+}\right)_{2}$. This is the most mobile of the two secondary ions appearing between $\mathrm{A}^{+}$and $(\mathrm{AB}) \mathrm{A}^{+}$in Figure 2, which we have interpreted as doubly charged clusters having fragmented between the DMA and the MS. Note that the mass assignment to all the $(\mathrm{AB})_{\mathrm{n}}\left(\mathrm{A}^{+}\right)_{2}$ for $\mathrm{n}<7$ (including $\left.(\mathrm{AB})_{2}\left(\mathrm{~A}^{+}\right)_{2}\right)$ is not based on direct mass spectrometric evidence, but on the principle of continuity used in [14]. It is striking that the two unstable clusters $(\mathrm{AB})_{2}\left(\mathrm{~A}^{+}\right)_{2}$ and $(\mathrm{AB})_{3}\left(\mathrm{~A}^{+}\right)_{2}$ would break up symmetrically by simultaneous loss of two $\mathrm{A}^{+}$ ions.

\section{Multiply Charged Clusters}

Figure 2 exhibits a broad background of small unresolved peaks in the region between $(\mathrm{AB}) \mathrm{A}^{+}$and $(\mathrm{AB})_{4} \mathrm{~A}^{+}$. This is the region previously identified in [14] (via DMA and charge reduction) as associated to multiply charged clusters with moderate charge states $\mathrm{z}=$ $\{2,3, \ldots 9, \ldots\}$ formed as dry residues from daughter drops having undergone repeated Coulomb explosions. This is the domain of our present focus. Preliminary analysis of this mobility region indicated the existence of a vast number of peaks, some corresponding neither to the $(\mathrm{AB})_{\mathrm{n}} \mathrm{A}^{+}$nor the $(\mathrm{AB})_{\mathrm{n}} \mathrm{NH}_{4}^{+}$series. Hence, the already small signal available is widely dispersed over the whole mass range, and it is rather difficult to infer anything meaningful from two-dimensional spectra $(Z$, $\mathrm{m} / \mathrm{z}$ ) such as those shown in Figure 2. However, a few initial mass spectra accumulating data for relatively long times at a fixed DMA setting revealed a rather interesting series of modulations in $\mathrm{m} / \mathrm{z}$ space, in many ways similar to that reported in reference [15] for the mobility distribution of charge-reduced clusters. In an effort to reduce the large data accumulation times necessary for these modulated structures to emerge above the background, we increased the solution concentration 10-fold to $0.2 \mathrm{M}$ in formamide (same salt). All subsequent data are for that highly concentrated solution. Table 3 lists the DMA voltages and corresponding mobilities $\mathrm{Z}$ at which mass spectra were accumulated. Measured DMA voltages were related to mobilities in this case using as a reference the value reported in [14] for the monomer ion $\mathrm{A}^{+}\left(0.96 \mathrm{~cm}^{2} \mathrm{~V} / \mathrm{s}\right)$. This reference was used before we noticed the discrepancy between the mobilities of this ion obtained in [14] and here, respectively. We have nonetheless used the earlier standard because of our belief that this is the right mobility when electrospraying from formamide solutions. The correctness of this choice is confirmed by the coincidence between the mobilities of the doubly charged ions identified in this series and those discussed in point $\mathrm{B}$ above.

Figure 3a shows the mass spectrum for a DMA voltage of $1550 \mathrm{~V}\left(1 / \mathrm{Z}=2.18 \mathrm{Vs} / \mathrm{cm}^{2}\right)$. This spectrum was accumulated for $192 \mathrm{~min}$ and shows with particular clarity several key features to be used in the interpretation of other similar spectra, taken at other mobilities over considerably shorter periods. One can see several sharp, widely dispersed peaks corresponding to singly charged ions (labeled $\left.\mathrm{n}^{+}\right)$. All these peaks $\left(0^{+}\right.$to $\left.5^{+}\right)$ appear at the same mobility, and are likely fragments of some of the very highly charged clusters selected by the DMA. Although apparently in relatively high abundance when judged by the peak height, the total charge carried by these fragments is much smaller than the sum charge carried by all of the higher charged clusters in the spectrum, and their relative abundance is therefore very small.

The only significant doubly charged ion at this

Table 3. Cubic root of cluster mass (Dalton ${ }^{1 / 3}$ ) at the crest of each modulation for the various mobilities (or DMA voltages) used, as a function of charge state (modulation number)

\begin{tabular}{|c|c|c|c|c|c|c|c|c|}
\hline $1 / \mathrm{Z}\left(\mathrm{Vs} / \mathrm{cm}^{2}\right)$ & 1.93 & 2.02 & 2.08 & 2.125 & 2.18 & 2.26 & 2.32 & 2.41 \\
\hline z\ V(Volt) & 1380 & 1440 & 1480 & 1510 & 1550 & 1600 & 1640 & 1700 \\
\hline 2 & & 19.31 & & & 17.36 & 17.41 & 17.9 & \\
\hline 3 & 22.5 & 22.95 & & 20.86 & 20.86 & 21.59 & 22 & 22.66 \\
\hline 4 & 25.89 & 26.59 & 24.55 & 24.66 & 25.16 & 25.65 & 26.1 & 26.58 \\
\hline 5 & 28.5 & 29.32 & 27.59 & 27.86 & 28.24 & 28.76 & 29.24 & 29.8 \\
\hline 6 & 31.18 & 32.15 & 30.416 & 30.68 & 31.12 & 31.8 & 32.28 & 33.23 \\
\hline 7 & 33.83 & 34.74 & 33.27 & 33.6 & 34.03 & 34.65 & 35.15 & 35.93 \\
\hline 8 & & 37.05 & 35.92 & 36.14 & 36.45 & 37.09 & 37.41 & 38.46 \\
\hline 9 & & & 38.39 & 38.56 & 38.93 & 39.44 & 40 & 40.99 \\
\hline 10 & & & 40.57 & 40.86 & 41.32 & 41.87 & 42.41 & 43.38 \\
\hline 11 & & & 42.54 & 42.97 & 43.44 & 44.22 & 44.72 & 45.76 \\
\hline 12 & & & 44.81 & 45.17 & 45.47 & 46.36 & 46.88 & \\
\hline 13 & & & 46.83 & 47.06 & & 48.24 & 49.26 & 50.13 \\
\hline 14 & & & 48.87 & 48.99 & & & 51.16 & \\
\hline 15 & & & & 50.94 & & & & 53.88 \\
\hline 16 & & & & 53.16 & & & & 56.01 \\
\hline
\end{tabular}



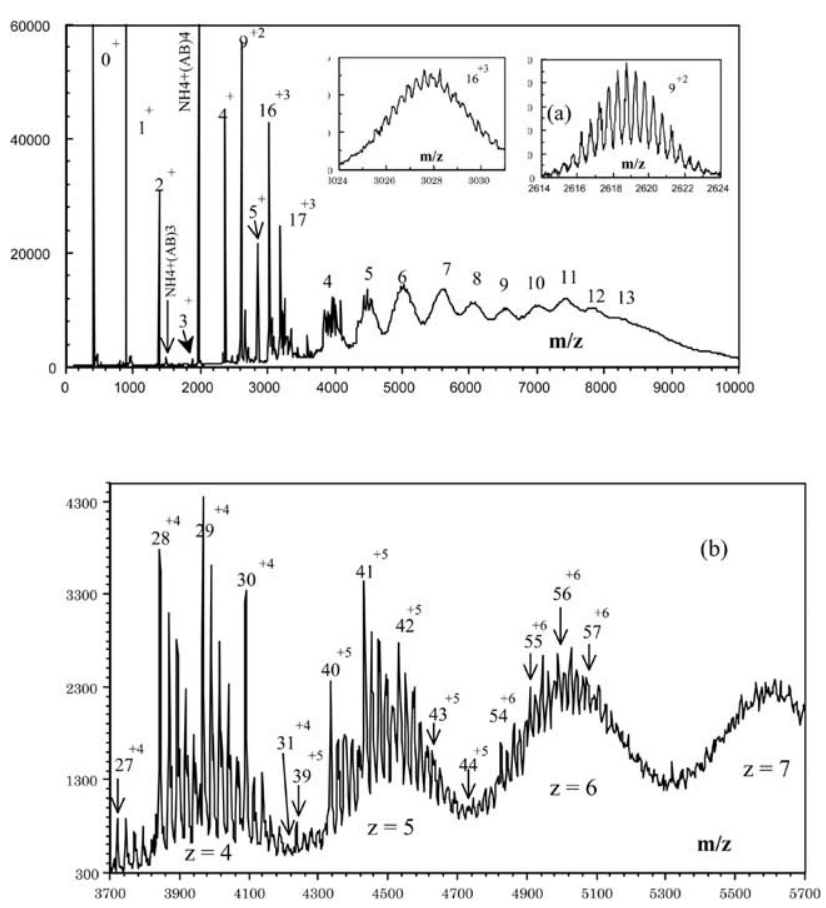

Figure 3. Mobility-selected MS spectrum at $1 / \mathrm{Z}=2.18 \mathrm{Vs} / \mathrm{cm}^{2}$ : (a) full spectrum; (b) close up on modulations $4-7 . \mathrm{n}^{+\mathrm{z}}$ stands for $(\mathrm{AB})_{\mathrm{n}}\left(\mathrm{A}^{+}\right)_{\mathrm{z}}$.

mobility, $(\mathrm{AB})_{9}\left(\mathrm{~A}^{+}\right)_{2}$ (labeled $9^{+2}$ ), is followed by a tail of smaller peaks, half of which can be identified as a series of adducts with one, two, three, $\ldots \mathrm{NH}_{4} \mathrm{Br}$ molecules. The other prominent members of this doubly charged tail correspond to multiple additions of an unidentified mass of approximately $124 \mathrm{u}$. All these secondary peaks can be unambiguously identified as doubly charged through the half mass unit spacing between neighboring isotopic peaks (see inset to Figure 3a). The same procedure confirms that the peak labeled $5^{+}$is mainly singly charged, with at most a few percent contribution of $10^{+2}$ buried within the noise. Two triply charged ions $\left(16^{+3}\right.$ and $\left.17^{+3}\right)$ from the main cluster series are present, both followed by a tail of triply charged adducts of the same composition as those trailing $9^{+2} \cdot 16^{+3}$ has sufficient signal to allow charge assignment based on isotopic spacing (see inset to Figure 3a), but this is no longer the case for either its adducts or $17^{+3}$, nor for any of the peaks with four or more charges. However, the intensity of the adducts, almost absent from singly charged ions, becomes increasingly important at higher $z$. The mass interval between such adduct ions and the bare salt clusters serves to confirm the charge state of the bare ions and their tail for $\mathrm{z}=3$ and beyond. Note that the mass of $\mathrm{AB}$ is nearly equal to five times the mass of $\mathrm{NH}_{4} \mathrm{Br}$, so that the sequence of $\mathrm{NH}_{4} \mathrm{Br}$ adducts goes only up to the fourth, the fifth being overwhelmed by the next bare cluster.

Figure $3 \mathrm{~b}$ shows a higher resolution detail of modulations 4 to 7 , with indication of the $(\mathrm{AB})_{\mathrm{n}}\left(\mathrm{A}^{+}\right)_{\mathrm{z}}$ clusters that can be identified. They are followed to their right by the usual series of adducts in the same charge state as the neat clusters, although the two series of adducts peaks can be distinguished fully only up to the fifth modulation. The sixth modulation is the last where well-defined ion peaks rise above the background and can be identified unambiguously. One still sees the sequence of four main adducted peaks following each clean cluster ion. But no clear distinction is now possible between the two kinds of adducts, while the bare ions do not dominate the landscape any more.

In conclusion, up to at least the sixth wave, the charge state of the ions under it is in all cases equal to the modulation number. This is exactly as seen in [13], where positive identification of charge states $\mathrm{z}$ and modulation number up to the ninth was possible, though without mass analysis. The unusual modulated structure observed both here and in earlier mobility studies, together with its straightforward explanation given in $[14,15]$ in terms of ion evaporation dynamics, justify extrapolating to higher modulations the identification between their order from left to right and the charge state $\mathrm{z}$. We further proceed to show that this extrapolation is strongly supported by the agreement found between the expected and the observed relation between mobility and mass.

\section{Relation Between Mobility, Mass, and Cluster Diameter}

For each mobility-selected mass spectrum obtained at each of the DMA settings listed in Table 3, positive ion identification of the initial modulations is carried out as discussed for Figure $3 a$ and $b$, and the subsequent waves are numbered sequentially as far as they can be identified unambiguously. In some cases where the $\mathrm{m}^{\text {th }}$ modulation was not clearly defined but subsequent ones were, the unclear datum was left undefined, but the subsequent well-defined peaks are included in the Table. In such incomplete series, the principle of continuity of the $\mathrm{m} / \mathrm{z}$ versus $\mathrm{z}$ curve was used to make sure that the $\mathrm{z}$ assignment was correct. Several associated mass spectra are shown in Figure 4 . They are noisier than in Figure 3 because they were accumulated for much shorter times (typically $10 \mathrm{~min}$ versus $190 \mathrm{~min}$ in Figure 3). The mass spectra shown in Figure 4 are at the maximum resolution at which they can be represented by the data acquisition routine. Mean $\mathrm{m} / \mathrm{z}$ values at the peaks were inferred more clearly by using wider bin sizes. The $\mathrm{m} / \mathrm{z}$ values at the center of each wave can be inferred from the $\mathrm{m}^{1 / 3}$ data listed in Table 3 as a function of modulation number $\mathrm{z}$ and inverse mobility $1 / Z$. We now note that the mobility of a sphere with diameter $\mathrm{d}$ in the free-molecule range is given by eq 1 $[19,20]$, where the subscript $E$ is introduced as a reminder that this is just a model of limited generality:

$$
Z_{E}=\frac{\beta z}{\left(d+d_{g}\right)^{2}}
$$



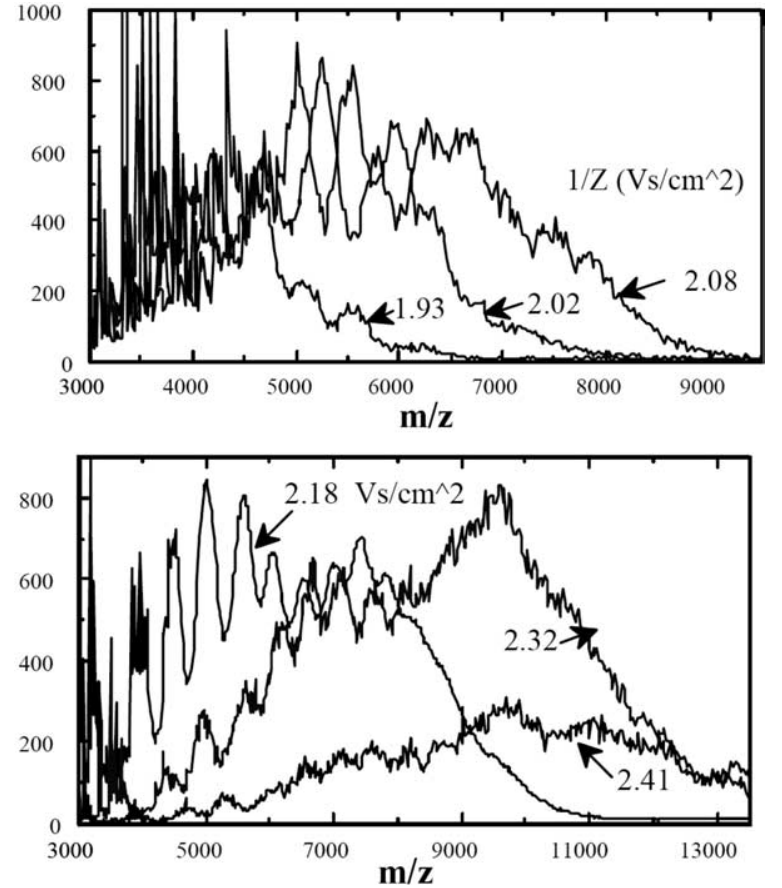

Figure 4. Z-selected mass spectra similar to those of Figure 3a obtained at other mobilities $\left(1.93 ; 2.02\right.$, and $2.08 \mathrm{Vs} / \mathrm{cm}^{2}$ at top, and $2.18,2.32$, and $2.41 \mathrm{Vs} / \mathrm{cm}^{2}$ at bottom).

where, for aerosol particles in air, the coefficient $\beta$ is given by [21]

$$
\begin{aligned}
& \beta=0.441 \frac{\mathrm{e}(\mathrm{kT} / \mu)^{1 / 2}}{p} \\
& \mu=\frac{\mathrm{mm}_{\mathrm{g}}}{\mathrm{m}+\mathrm{m}_{\mathrm{g}}}
\end{aligned}
$$

while $\mathrm{p}, \mathrm{m}_{\mathrm{g}}$, and $\mathrm{T}$ are the gas pressure, mass and absolute temperature; $\mathrm{k}$ is Boltzmann's constant and $\mathrm{q}$ $=$ ze is the electrical charge on the particle. $\mathrm{m}$ is the particle mass, and $d_{g}$ the effective diameter of the colliding gas molecules. Note that the numerical value 0.441 appearing in eq 2 corresponds to almost inelastic gas-particle collisions, and would be a factor 1.357 times larger if the collisions were elastic. When drawing experimental cross sections from mobility measurements, chemists tend to assume that collisions are elastic. Both hypotheses cannot evidently be right over the whole size range going from micron drops to nanometer or subnanometer clusters. The value 0.441 is adequately established for gas particle collisions, which tend to be close to inelastic at particle diameters of tens of $\mathrm{nm}$. The alternative elastic assumption applies surely to atom-atom collisions at room temperature, and perhaps also to collisions between atoms or small molecules and relatively small clusters. Since the masses represented in Figure 5 go from 5000 to beyond 175,000 u (Table 3), the inelastic behavior implicit in eq 2 appears as adequate (however, see below). We note, nonetheless, that all previous studies using mobility-MS analysis have implicitly assumed that collisions with $\mathrm{He}$ atoms are elastic [22], even for rather large species such as proteins. In contrast, Tammet [19] has discussed the conditions under which transition from elastic to inelastic collisions would take place as the number of degrees of freedom increase, and he places it approximately at a cluster diameter of $2 \mathrm{~nm}$.

If the bulk density of the material composing the particle is $\rho$ and its value is not changed by the high curvature, we may relate the particle mass and diameter via:

$$
\mathrm{d}^{3}=6 \mathrm{~m} /(\pi \rho)
$$

It follows that $\mathrm{m}^{1 / 3}$ and $(\mathrm{z} / \mathrm{Z})^{1 / 2}$ are both linear with particle diameter, and hence linear with each other. Elimination of $d$ between eq 2 and eq 4 then yields the following relation between $\mathrm{Z}$ and $\mathrm{m}$ :

$$
\mathrm{m}^{1 / 3}+\mathrm{m}_{0}^{1 / 3}=(\rho \pi / 6)^{1 / 3}(\beta \mathrm{z} / \mathrm{Z})^{1 / 2},
$$

where we have defined the auxiliary quantity $\mathrm{m}_{\mathrm{o}}$ :

$$
\mathrm{m}_{\mathrm{o}}=\rho \pi \mathrm{d}_{\mathrm{g}}{ }^{3 / 6},
$$

which is of the order of the mass of gas molecules (but should not be identified with it). Note that although the quantity $\beta$ depends on cluster mass $m$, the large mass disparity between the gas molecules and the clusters makes $\mu$ in eq 3 almost identical to the gas molecule mass, and hence $\beta$ is essentially constant for all clusters. Consequently, a plot of $\mathrm{m}^{1 / 3}$ versus $\mathrm{z}^{1 / 2}$ should give a straight line for all the clusters observed at a fixed mobility, with a slope simply related to the corresponding $\mathrm{Z}$ value. Alternatively, plots of $(\mathrm{z} / \mathrm{Z})^{1 / 2}$ versus $\mathrm{m}^{1 / 3}$

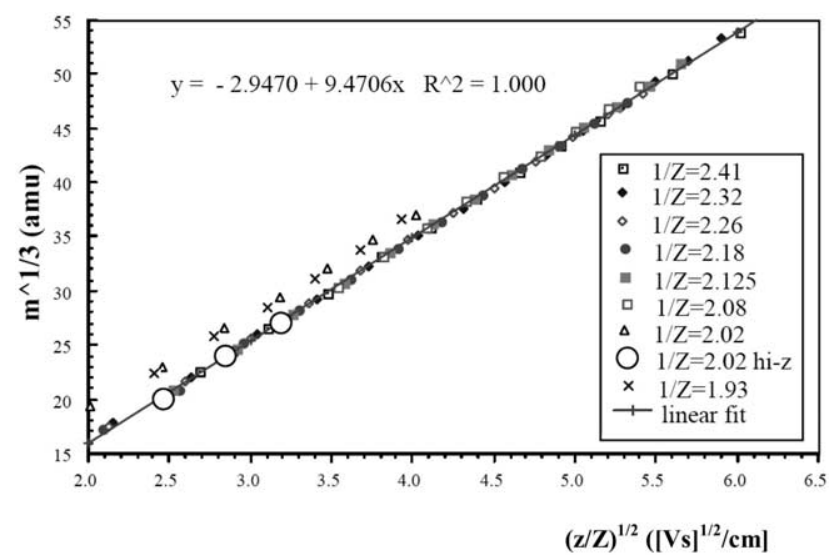

Figure 5. $\mathrm{m}^{1 / 3}$ versus $(\mathrm{Z} / \mathrm{z})^{1 / 2}$ plots for all the Z-selected mass spectra taken. The anomalous position of the data for the two highest mobilities is due to charge loss between the DMA and the MS. The large open circles (labeled $1 / \mathrm{Z}=2.2 \mathrm{Vs} / \mathrm{cm}^{2}$ high-z) are from the unconverted fraction of these metastable ions (see Figure 6). 
should give a unique straight line at all mobilities. As shown in Figure 5, this is in fact the case for almost all data points. The exceptions correspond to the clusters with the two largest mobilities $(1 / \mathrm{Z}=2.02$ and $1 / \mathrm{Z}=$ 1.93). A clue to the anomalous behavior can be obtained by examining Figure 6, the mass spectrum for $1 / \mathrm{Z}=$ 2.02. In contrast to the lower mobility spectra (exemplified by Figure 3 for $1 / Z=2.18$ ), there are two series of overlapping charge states that appear to complicate the pattern. For example, there is a series of $4+$ peaks at approximately $m / z 4500-4800$, and a series of $4+$ peaks centered at $\mathrm{m} / \mathrm{z}$ 3500. There are similar pairs indicated in Figure 6 for the $3+$ and $5+$ series. The two sets of peaks of the same charge but different $m / z$ must either be two widely different conformations of the same ions, or else the lower $\mathrm{m} / \mathrm{z}$ ions must be daughters from larger ions having fragmented between the DMA and the MS. It is unlikely that different conformations of such large ions exist with such shape differences, so the latter explanation seems most likely. Thus, the $2+$ ions around $\mathrm{m} / \mathrm{z}$ 3000 are probably fragments of the $3+$ species at $\mathrm{m} / \mathrm{z}$ 2700; the $3+$ ions around $m / z 4250$ are fragments of the $4+$ peak at $m / z$ 3500; and the $4+$ ions at $m / z 4500-4800$ are fragments of the $5+$ ions around $m / z 4000$. The mass and charge differences appear to correspond approximately to the loss of $(\mathrm{AB})_{2} \mathrm{~A}^{+}$. This inference from comparing the two series in Figure 6 is also in qualitative agreement with the mass spectra (not shown) for the data in the inset of Figure 2. The mass distribution for the large clusters under the mobility peak at 3.2 $\mathrm{Vs} / \mathrm{cm}^{2}$ includes preferentially the $(\mathrm{AB}) \mathrm{A}^{+}$cluster, with about half the intensity of $\mathrm{A}^{+}$and $(\mathrm{AB})_{2} \mathrm{~A}^{+}$. Such a charge loss mechanism, akin to ion evaporation from the dry residue, has been observed previously in tandem DMA experiments [13] at atmospheric pressure.

No precursor series for the $6+$ ions and higher at $m / z$ $>4000$ are observed. The only explanation is that the charge loss [of $(\mathrm{AB})_{2} \mathrm{~A}^{+}$] is complete for the higher charged clusters before they reach the MS, or else in the MS interface itself. We propose that this loss of one singly charged ion between the DMA and the MS occurs for only the two highest mobility series, and is complete except for the $3+, 4+$, and $5+$ series where only partial conversion arises. This explanation is strongly supported by the data of Figure 5, which identified the two anomalous series because the points do not fall on the same line as all of the other series. Replotting the anomalous points with their mass and charge modified by respective addition of the mass and charge of the presumed fragment lost $\left[(\mathrm{AB})_{2} \mathrm{~A}^{+}\right]$brings them right on the main line (corrected points not shown). The three points corresponding to the lower $\mathrm{m} / \mathrm{z}$ values (charge states 3,4 , and 5 ) are also plotted in Figure 6 (large open circles). They fall right on the line, strongly supporting the explanation of charge loss.

The reason why the $\mathrm{z}=3,4$, and 5 charge states at $1 / \mathrm{Z}=2.02 \mathrm{Vs} / \mathrm{cm}^{2}$ survive in part in going from the DMA to the MS, yet none of the higher charge states remain in their original state at high $Z$, can be under- stood in light of previous work [13, 23]. The ion evaporation mechanism from drops is controlled for large drops mainly by the electric field $\mathrm{E}$ on their surface. Hence, a large drop that evaporates solvent and ions will tend to evolve at a constant electric field E. However, the increasing curvature effects arising in the rather small size range investigated here tend to require increasing electric fields to evaporate ions from the smallest drops [14]. Consequently, when these drops evaporate completely, leaving behind a charged residue, the surface electric field can be very high, and it increases with decreasing cluster mass. Since Z and E are closely related to each other, it makes sense that the most mobile clusters having the highest surface electric field are metastable toward further evaporation of an ion from the dry residue. In addition, at a fixed $Z$ (such as in each of our MS spectra), curvature effects are different for the various cluster sizes, and one would expect some of them would tend to evaporate ions more readily than others. This explains why at $1 / Z=2.02$, charge states higher than $6^{+}$have completely lost one ion, while those at $3^{+}, 4^{+}$, and $5^{+}$are only partly converted. What our data show is that the critical curves for ion evaporation from a liquid and a solid are such that the former phenomenon occurs more easily than the second at larger diameters, but the opposite is true at smaller diameters, where ions continue to evaporate after the particle becomes solid. The crossing point appears to be only a function of mobility for the larger diameters (hence a function only of E). But it has a more complex dependence on $\mathrm{E}$ and $\mathrm{R}$ at smaller sizes, and the curvature effect on the activation energy for ion evaporation seems to be different from drops and from solids.

For all the clusters investigated, we now have reliable values not only for $\mathrm{m}$ and $\mathrm{z}$, but also a fairly robust procedure to determine their diameter. Indeed, the relation between $\mathrm{m}$ and $\mathrm{Z}$ experimentally established in Figure 5 gives a slope and an intersection with the vertical axis, from which the following values for the quantities $\mathrm{d}_{\mathrm{g}}$ and $\rho$ follow:

$$
\begin{aligned}
& \mathrm{d}_{\mathrm{g}}=0.443 \mathrm{~nm} \\
& \rho=0.935 \mathrm{~g} / \mathrm{cm}^{3} .
\end{aligned}
$$

There are several sources of ambiguity in this inference. One could be associated to an inappropriate value for the coefficient 0.441 used in eq 2, as discussed following eqs 1-3. A second source of error associated with the use of eq 2 and eq 4 to infer diameter from mass would arise if the clusters were nonspherical. If all had the same shape (say cubes), this would merely force a reinterpretation of the density obtained from the slope as an effective rather than actual density. The real density would coincide with the effective value only for spheres, and would otherwise exceed it by a packing factor easily calculated for symmetric shapes, for which the mobility is strictly proportional to wetted area [18]. 


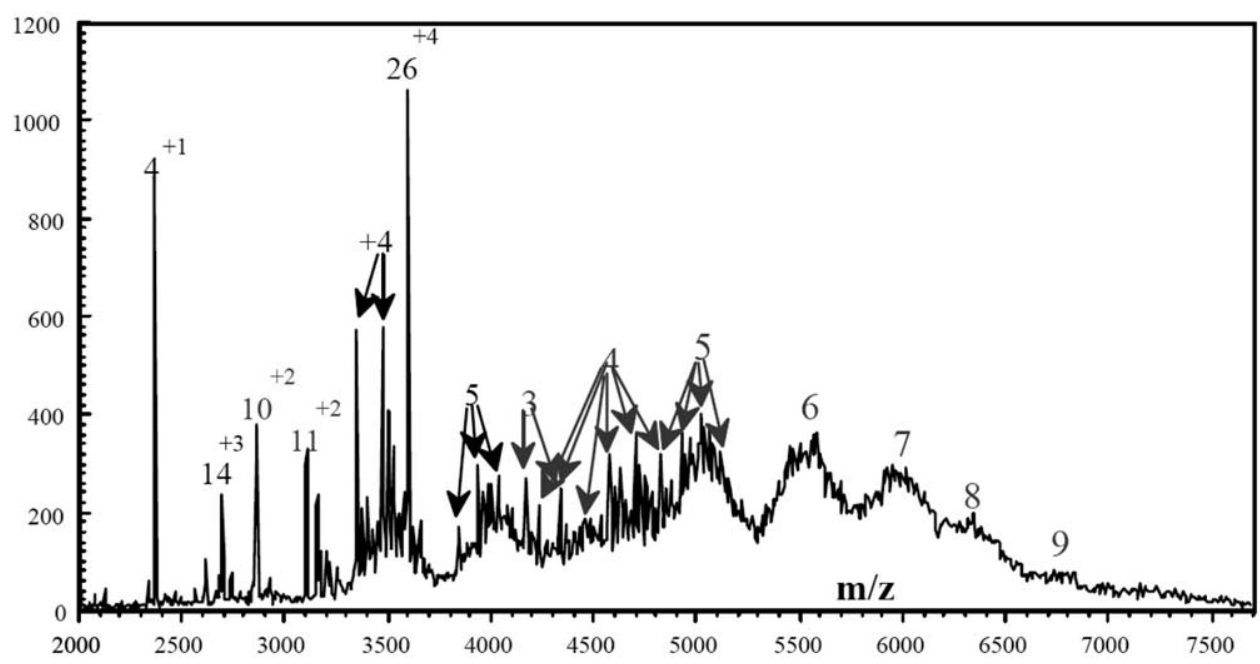

Figure 6. Full mass spectrum for $1 / \mathrm{Z}=2.02 \mathrm{Vs} / \mathrm{cm}^{2}$. The peaks marked $10^{+2}$ and $11^{+2}$, and the modulations to the right of the $26^{+4}$ peak marked 3-9, all have anomalously low charge states corresponding to metastable clusters having lost one charge between the DMA and the MS. Other marked groups are in their original charge state.

For cubic clusters, the correction to the density would be 1.383 , bringing $\rho$ to a value of $1.293 \mathrm{~g} / \mathrm{cc}$. We are not aware of data for the density of tetraheptyl ammonium bromide. Values for the analogous tetramethyl and tetraethyl salts are 1.56 and $1.397 \mathrm{~g} / \mathrm{cm}^{3}$. This rapidly decreasing $\rho$ at increasing alkyl chain length should continue, since in the limit of a very long chain, one would presumably recover the density of a long alkyl chain $\left[0.807 \mathrm{~g} / \mathrm{cm}^{3}\right.$ for solid $\mathrm{CH}_{3}\left(\mathrm{CH}_{2}\right)_{26} \mathrm{CH}_{3}$ at room temperature]. An upper limit estimate of the density of our salt can be made by taking the density of the alkyl component to be $0.807 \mathrm{~g} / \mathrm{cc}$, and accounting for the densification associated to the ammonium group by simply adding the volumes of three alkanes and one alkyl amine groups with the latter being 1.135 more dense than the former (as in liquid heptane and heptyl amine at room temperature). The resulting density ignoring the volume of bromine but including its mass is $0.998 \mathrm{~g} / \mathrm{cm}^{3}$. This upper limit exceeds that inferred from the slope of Figure 5 by just $6.4 \%$, confirming the notion that the clusters are fairly compact. Similar studies [24] with singly charged polyethylene glycol (PEG) clusters in the mass range from 4000 to 700,000 have reported a cluster density of $1.23 \mathrm{~g} / \mathrm{cc}$, in fair agreement with the known density of PEGs with masses above a few thousand ( $\rho_{\mathrm{PEG}}=1.21 \mathrm{~g} / \mathrm{cc}$ [24]), apparently confirming the near spherical shape of these clusters. This study, however, did not measure ion mass directly, while a subsequent DMA-MS investigation has inferred the smaller value $\rho_{\mathrm{PEG}}=1.055 \mathrm{~g} / \mathrm{cc}$ [25]. Although the difference is substantial, it would only alter the particle diameter by $5 \%$. A similar DMA-MS study with singly charged polystyrene ions has found ion densities very close to the bulk, and speculated that the low PEG densities observed could be caused by the softness of this material, due to the proximity between its glass transition point and room temperature [25].
A third source of errors in the size versus mobility relation (eq 2) would be due to the long range interaction with the dipoles induced in the bath gas molecules by the multiple charges on the clusters. This correction is a function of the ratio of the polarization energy at contact over $\mathrm{kT}$, which is strictly proportional to $\mathrm{z} /\left(\mathrm{d}+\mathrm{d}_{\mathrm{g}}\right)^{2}$ [26]. Hence, at fixed temperature and carrier gas, the polarization correction is exactly the same for all clusters of the same mobility, independently of their charge state. Therefore, all the clusters in each of the mobility-selected series investigated have the same mobility as well as exactly the same $\mathrm{z} /\left(\mathrm{d}+\mathrm{d}_{\mathrm{g}}\right)^{2}$ value:

$$
\mathrm{Z}=\mathrm{F}\left(\mathrm{Z}_{\mathrm{E}}\right)
$$

The curves shown in Figure 5 would then be expected to remain linear, but the mobility would no longer be exactly proportional to the right hand side of eq 2 . Instead, it would be a function of this right hand side, very close to it at small mobilities (small polarization effect), but departing from it at higher mobilities. Consequently, the various curves shown in Figure 5 would not collapse into each other in terms of the variable $(\mathrm{z} / \mathrm{Z})^{1 / 2}$, and the slight differences observed would yield the polarization effect on $Z$.

Some departure of the main trend of Figure 5 can in fact be clearly seen in Figure 7, where we include our new data for singly and doubly charged particles, as well as those reported in [13] (with the corrected mass assignment). Comparison of the singly (filled circles) and doubly charged series (open circles) shows that, at a fixed mass (size), an increased charge increases slightly the drag, pointing directly to a polarization effect. Although this effect tends to die out at increasing masses, it is not clear that it has disappeared entirely in the mobility range of the multiply charged clusters.

Another effect opposite to polarization can also be 

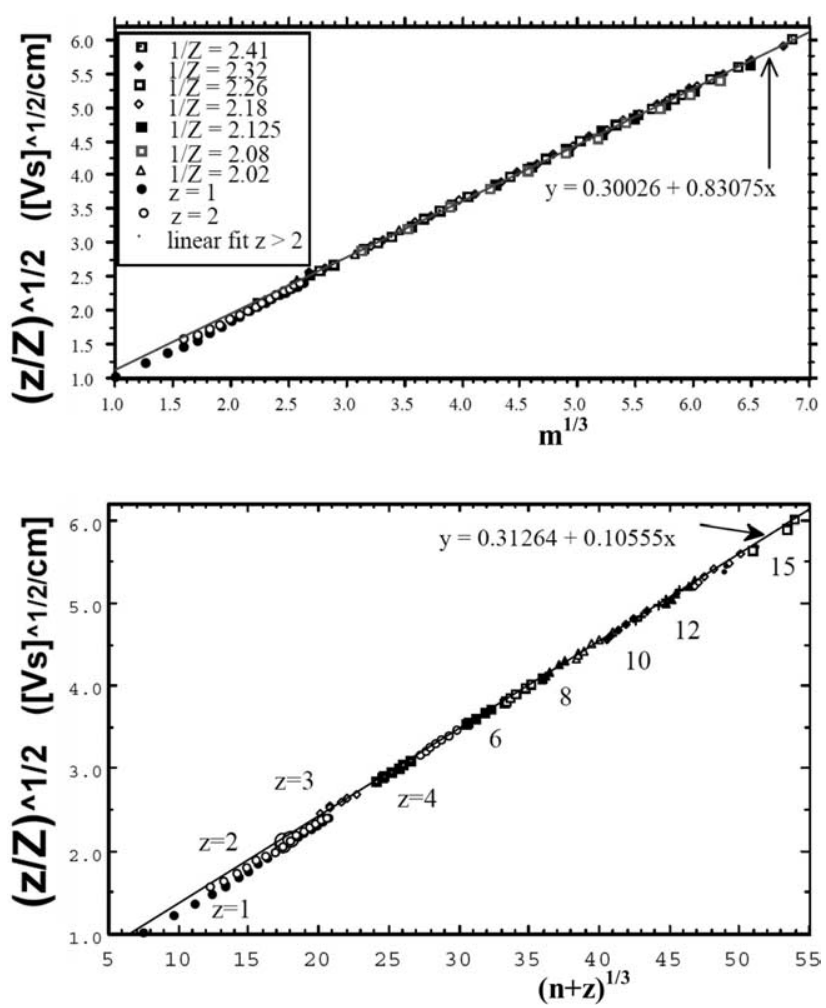

Figure 7. Square root of the inverse mobility versus the cluster size, measured in (a) by the $1 / 3$ power of cluster mass, and in (b) by the $1 / 3$ power of the number of cations $(n+z)$ in the cluster. The figures include naturally charged clusters as well as the wider series of the singly and doubly charged ions, many of which are from reference [13] and are charge-reduced. The data for singly and doubly charged clusters fall clearly below the main trend and differ from each other.

seen in the figure: The singly and doubly charged clusters fall clearly below the main trend line, indicating that they are anomalously mobile. This trend is in fact expected from the known tendency of very small clusters to reach densities larger than the bulk, as well as from the tendency for the collisions with the background gas to become more elastic [19]. What is surprising is the fact that these phenomena set in at such relatively large sizes and dominate initially over the opposing polarization effect. We note that the calibrations setting the mobility scales are identical for the singly and doubly charged series, but they differ from those leading to the data of Figure 5. The differences cannot be large, since the three doubly charged data points in the multiply charged data series (large open circles in Figure 7) fall very close to the doubly charged series (though two of them are slightly higher). Furthermore, the singly and doubly charged data curve downwards in Figure 7, while polarization effects alone would curve them upwards.

These anomalies are slight, but they are very worth pursuing because the possibility to infer accurate values of the density of such large clusters via mobility measurements is of fundamental interest. For this reason we have made an effort to isolate the polarization effects from those increasing the mobility of the smallest clusters. One possible interfering factor is the fact that the density of the clusters decreases at smaller sizes since the fraction of bromine they carry diminishes. Particle volume would evidently be a better measure of its diameter than mass, and because volume is dominated by the cations, we have represented in Figure $7 \mathrm{~b}$ the data of Figure $7 \mathrm{a}$ in terms of the $1 / 3$ power of the number of cations $(n+z)^{1 / 3}$ in $(A B)_{n}\left(A^{+}\right)_{z}$. The effects seen in Figure $7 \mathrm{a}$ remain in Figure $7 \mathrm{~b}$.

Polarization effects can be isolated by reference to eq $7 \mathrm{c}$, though with the warning that this formula can be expected to hold only at large enough sizes, where collisions are still inelastic and the density is still close to the bulk. The inverse of eq $7 \mathrm{c}$ can be written $(\mathrm{d}+$ $\left.d_{g}\right) / \sqrt{ } z=G(Z)$, which in turn implies that $(n+z)^{1 / 3}$ should be linear with $\sqrt{ } z$, with a slope that should depend on $Z$ and an intercept (at zero $V \mathbf{z}$ ) that should not. Linear fits of such lines for fixed $Z$ (data in Table 3 ) show that they are very closely linear, with slight changes in the intercept. Conditioning the determination of the regression slope by forcing this intercept to be the same for all $Z$ values yields the function $G(Z)$, with values almost exactly proportional to $1 / \sqrt{ } Z$ $[\mathrm{G}(\mathrm{Z}) \sqrt{ } \mathrm{Z}=1.193,1.195,1.192,1.194,1.1998,1.204$, and 1.194 for decreasing $1 / Z$ from 2.41 to $2.20 \mathrm{Vs} / \mathrm{cm}^{2}$ ]. The common intercept yielding a best fit is at $(n+z)^{1 / 3}=$ 0.33 . Consequently, eq $7 \mathrm{c}$ accounting for polarization can be written in the form

$$
1 / \sqrt{Z}=F\left[\left((n+z)^{1 / 3}+0.33\right) / \sqrt{z}\right]
$$

This representation is used in Figure 8, showing that all the data of Table 3 for the multiply charged clusters collapse together. Doubly charged clusters (filled triangles) are below the main line by about $2 \%$, and singly charged clusters (filled circles) by about $8 \%$. We now

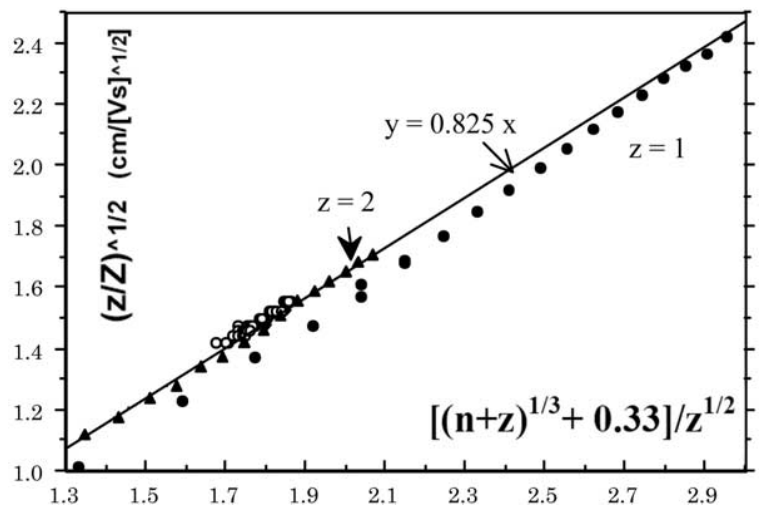

Figure 8. Representation of the data of Figure 7 in terms of the polarization parameter $(\mathrm{d}+\mathrm{dg}) / \sqrt{ } \mathrm{z}$, where the particle diameter is represented by $(n+z)^{1 / 3}$, and is shifted by the associated effective gas molecule diameter (0.33). The behavior at small $\mathrm{Z}$ (top right corner, with negligible polarization effect) should tend to a straight line through the origin. It is represented by the line $y$ $=0.825 \times$ to which the singly and doubly charged particle series (and the multiply charged cloud) asymptote. 
use the fact that for large particles there is no polarization effect, so that the function $\mathrm{F}$ in eq $7 \mathrm{~d}$ should be a straight line through the origin. By drawing one line through the origin and the datum with the smallest $\mathrm{Z}$ (the largest singly charged particles), one sees indeed that the singly charged series appears to asymptote to it, as expected. Curiously, the doubly charged data do also go through this asymptote, though this must be a coincidence associated with the fact that the factor tending to increase the mobility cancels approximately the contrary effect of the ion-dipole interaction. The cloud of cluster data falls clearly above this asymptote (though approaching it at increasing $1 / \sqrt{ } \mathrm{Z}$ ), implying that polarization is small, but not negligible, even at these relatively low mobilities. The "extra" effects leading to an increase in mobility in the singly and doubly charged ions can be measured initially as the departure from the straight line asymptote, and then from their departure from the slight trend to curve upwards defined by the larger clusters. The tendency is very clear in the case of the singly charged series, as it begins at mobilities small enough for polarization effects to be almost entirely absent. The anomalous increase in mobility sets in at a value of the ordinate of about $2.8(\mathrm{~m} \sim$ $8000 \mathrm{Da} ; \mathrm{d} \sim 3 \mathrm{~nm}$ ), and saturates shortly after. If this were strictly a size effect (as expected both for densification and for a decrease in the inelasticity of collisions), then the same effect would be expected on the doubly charged clusters at a value of the horizontal axis $\sqrt{ } 2$ times smaller than 2.8 (1.98). This is exactly what happens, though the phenomenon is obscured in this case because polarization effects now act concurrently, whereby the doubly charged series first curves downwards below the asymptote, and then curves upwards crossing it again. A complete transition from inelastic to elastic drag would increase the mobility by a factor of 1.36 , leading to a decrease of $16.6 \%$ in $1 / \sqrt{ } \mathrm{Z}$. Only half of this effect is seen in Figure 8.

Although our measurements are sufficiently accurate to demonstrate unambiguously such qualitative trends, the calibration procedure for the mobility measurement was not adequate to quantify them at the precision level required to capture variations of just one or a few percent. We therefore conclude the discussion with the final remark that the effects observed here for the first time had been advanced by Tammet's [19] warning that the accommodation coefficient depends on particle size. This suggest a revision of the common hypothesis that the collisions are inelastic (or elastic) independently of ion size.

\section{Ion Evaporation Kinetics}

Having established that the relation between size mass and mobility embodied in eqs $2-7$ is reliable (within a $2 \%$ error), we use eq 4 and eq $7 b$ to turn all the $m(Z)$ data of Table 3 into a $\mathrm{d}(\mathrm{z})$ form suitable to infer charge and curvature effects on ion evaporation kinetics. All such data (with the exclusion of those at the two highest mobilities for which there is evidence of metastability) are shown as individual points in Figure 9. The spread of cluster diameters represented at each $\mathrm{z}$ is associated to the $\mathrm{Z}$ dependence of the center of the modulations. There is in reality an even greater spread, since each modulation has a certain width, but this will be ignored here because it does not modify the qualitative picture much, and would leave the center of the $(d, z)$ region populated by clusters virtually unchanged.

The interpretation of the data of Figure 9 in terms of ion evaporation kinetics has been discussed in detail previously [13], but can be summarized as follows. As a charged drop evaporates, it moves vertically in the $(z$, d) space of Figure 9a. It will eventually reach some critical condition at which it loses one charge almost instantly, and jumps almost horizontally one $\mathrm{z}$ unit to the left. By repetition of this process it will follow a staircase trajectory, limited from below by the critical curve $d_{\text {crit }}(z)$ at which the ion evaporates, and from above by this same curve displaced one charge unit to the left. Drops undergoing solvent and ion evaporation will then remain within these two critical curves, and so will the final residues into which they eventually dry. On the assumption that the clusters we observe are formed by this mechanism, their charge and diameter should lie within such critical curves. The critical curves measured experimentally can evidently be used to infer information on ion evaporation kinetics.

The exponent of the Iribarne-Thomson rate contains an activation energy $\Delta$ that depends on cluster charge and diameter [12]. A general form for this dependence may be derived under the assumption that the drop is perfectly conducting and remains spherical during ion evaporation, while the escaping ion behaves as a point charge [14, 27]

$$
\Delta=\Delta G_{\mathrm{S}}^{\mathrm{o}}-\frac{\mathrm{e}^{2}}{2 \pi \varepsilon_{\mathrm{o}} \mathrm{d}}[\mathrm{F}(\mathrm{z})+\alpha]
$$

where $\alpha$ is a dimensionless constant measuring the effect of curvature, $\Delta \mathrm{G}_{\mathrm{S}}^{\mathrm{o}}$ is the usual Gibbs free energy for ion evaporation from a planar uncharged liquid surface. $\mathrm{F}(\mathrm{z})$ is a known function, computed up to $\mathrm{z}=$ 10 in Table 4 of [14], and shown in Table 4 above at half unit intervals up to $\mathrm{z}=20$. At critical conditions, the activation energy ratio $\Delta^{*} / \mathrm{kT}$ is fixed by the requirement that the rate of drop evaporation matches that of ion evaporation, as given by eqs $19-20$ of [13]. Eq 8a hence fixes the following relation between critical cluster diameter and charge at which ion evaporation occurs:

$$
\mathrm{d}_{\text {crit }}(\mathrm{z}+1) / \mathrm{d}^{*}=\mathrm{F}(\mathrm{z})+\alpha,
$$

where the quantity $\mathrm{d}^{*}$ having units of length denotes the following combination of variables 

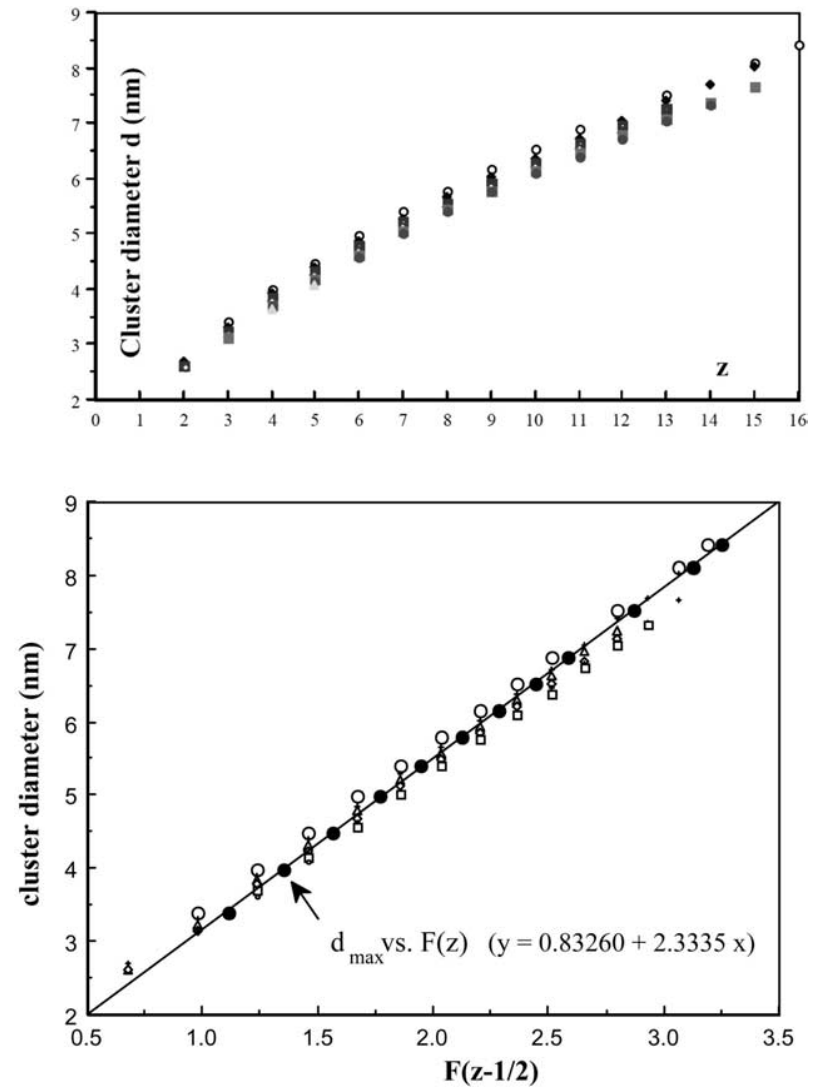

Figure 9. Cluster diameter for the various mobilities investigated plotted as a function of the charge state $\mathrm{z}(\mathbf{a})$, or the function $\mathrm{F}(\mathrm{z}-1 / 2)(\mathbf{b})$. The filled circles with a fitted line represent the data at the smallest mobility (open circles, $1 / \mathrm{Z}=2.41 \mathrm{Vs} / \mathrm{cm}^{2}$ ) versus $\mathrm{F}(\mathrm{z})$ rather than $\mathrm{F}(\mathrm{z}-1 / 2)$, and have been used to infer the parameters $\Delta \mathrm{G}_{\mathrm{S}}^{\mathrm{o}}$ and $\alpha$.

$$
\mathrm{d}^{*}=\frac{\mathrm{e}^{2}}{2 \pi \varepsilon_{\mathrm{o}}\left(\Delta \mathrm{G}_{\mathrm{S}}^{\mathrm{o}}-\Delta^{*}\right)} .
$$

Consequently, the predicted minimum diameter $\mathrm{d}_{\text {min }}(\mathrm{z})$ is $\mathrm{d}^{*}\left[(\mathrm{~F}(\mathrm{z}-1)+\alpha]\right.$, and its maximum value is $\mathrm{d}_{\max }=\mathrm{d}^{*}$ $[\mathrm{F}(\mathrm{z})+\alpha]$. One can see in Figure 9a that the width of the $(\mathrm{d}, \mathrm{z})$ domain populated by clusters does indeed span approximately one unit charge, as predicted. Regarding the shape of this domain's boundary, since it is easiest experimentally to determine the mean, instead of eq $8 b$ we will use first the equivalent but more convenient criterion:

$$
\mathrm{d}_{\text {mean }}(\mathrm{z})=\mathrm{d}^{*}[\mathrm{~F}(\mathrm{z}-1 / 2)+\alpha]
$$

In other words, representing the core data of Figure 9a in terms of the function $F(z-1 / 2)$ rather than $z$ should yield a straight line, with a slope equal to $\mathrm{d}^{*}$ and an intercept with the vertical axis equal to $\mathrm{d}^{*} \alpha$. Such curves are shown in Figure 9b. Whereas Figure 9a exhibits considerable curvature, the new representation yields indeed straight lines. A linear fit for the mobilityselected data falls in the middle yields $\mathrm{d}^{*}=2.16 \mathrm{~nm} ; \alpha$ $=1.1 / 2.16=0.509$. Reference [13] has obtained a similar value for $\mathrm{d}^{*}(2.15 \mathrm{~nm})$, but a considerably smaller $\alpha$ (0.325). A weakness of the interpretation based on $d_{\text {mean }}(z)$ is that the $(d, F)$ data are slanted towards the upper left corner of the graph because of the metastability of the smallest or most highly charged particles. This difficulty can be overcome here because the least mobile clusters are stable. Noting that $\mathrm{d}_{\text {crit }}(\mathrm{z}+$ $1) / d^{*}=d_{\min }(z+1) / d^{*}=d_{\max }(z) / d^{*}=F(z)+\alpha[14]$, we represent also in Figure $9 b$ (filled circles) the data on the least mobile particles as a function of $\mathrm{F}(\mathrm{z})$ [rather than as a function of $\mathrm{F}(\mathrm{z}-1 / 2)]$. The corresponding curve goes broadly through the middle of the data cloud, except at small $\mathrm{z}$, where the missing metastable data points would be expected to lie. We will therefore base the inference of $\mathrm{d}^{*}$ and $\alpha$ on these $\mathrm{d}_{\max }(\mathrm{z})$ data, whose linear fit in Figure $9 b$ gives:

$$
\begin{aligned}
& \mathrm{d}^{*}=2.33 \mathrm{~nm} \\
& \alpha=0.83260 / 2.33=0.357 .
\end{aligned}
$$

The new value of $\alpha$ is much closer to that of reference [13], but $\mathrm{d}^{*}$ is almost $8 \%$ larger. A discussion of which of the two results is to be trusted best is therefore warranted. One relevant observation is that [13] included considerably more data points than we do in the metastable region, evidently because their analysis (DMA, charge-reduction, DMA) happened all at atmospheric pressure, while the transit of our clusters into the vacuum system must be destroying the least stable among them. In contrast, we observe more clearly the clusters at low mobilities, almost down to the smallest $Z$ at which they can be seen in the spectrum of Figure 2a (inset). In particular, for each of these low Z, we can distinguish the modulations corresponding to fairly high $\mathrm{z}$ values. The probable reason for these advantages over [13] is the interference from the very large particles associated to the mobility peak at $3.2 \mathrm{Vs} / \mathrm{cm}^{2}$, whose tail extends into the low mobility tail of the cluster peaks under study. The charge on these large particles appears to be determined by the dynamics of Coulomb explosions rather than ion evaporation, and their $\mathrm{z}(\mathrm{d})$ relation therefore follows a different pattern. This inter-

Table 4. Ion evaporation function $\mathrm{F}(\mathrm{z})$

\begin{tabular}{lllllllllll}
\hline \multicolumn{1}{c}{$z$} & \multicolumn{1}{c}{1} & \multicolumn{1}{c}{2} & \multicolumn{1}{c}{3} & \multicolumn{1}{c}{4} & \multicolumn{1}{c}{5} & \multicolumn{1}{c}{6} & 7 & \multicolumn{1}{c}{8} & \multicolumn{1}{c}{9} \\
\hline \hline $\mathrm{F}(\mathrm{z}-1 / 2)$ & 0.2898 & 0.67778 & 0.97871 & 1.23491 & 1.46221 & 1.66876 & 1.8594 & 2.03745 & 2.20505 & 2.36389 \\
$\mathrm{~F}(\mathrm{z})$ & 0.5 & 0.8354 & 1.1111 & 1.3515 & 1.5677 & 1.7658 & 1.9498 & 2.1224 & 2.2855 & 2.4404 \\
$\mathrm{~F}(\mathrm{z}+9.5)$ & 2.5152 & 2.66003 & 2.79909 & 2.93304 & 3.06241 & 3.18764 & 3.3091 & 3.42712 & 3.54197 & 3.6539 \\
$\mathrm{~F}(\mathrm{z}+10)$ & 2.5884 & 2.7302 & 2.8667 & 2.9983 & 3.1255 & 3.2488 & 3.3685 & 3.4849 & 3.5983 & 3.7088 \\
\hline
\end{tabular}


ference is harder to overcome in a separation method based on mobility alone, but is automatically avoided here by the circumstance that the MS detector is insensitive to such large particles.

In conclusion, the data taken here at the smallest mobility are fairly representative of the largest particle diameters present at each $\mathrm{z}$, and should therefore satisfy the condition $\mathrm{d}_{\max }(\mathrm{z})=\mathrm{F}(\mathrm{z})+\alpha$ used to infer $\mathrm{d}^{*}$ and $\alpha$. In addition, these low $Z$ data are free from interference from metastable particles, and include relatively large $\mathrm{z}$ values that yield good regression slopes. We hence consider the present measurements more reliable than those previously available. Using eq 11 for $\mathrm{d}^{*}$, eq 9 implies

$$
\Delta \mathrm{G}_{\mathrm{S}}^{\mathrm{o}}-\Delta^{*}=1.23 \mathrm{eV} .
$$

The earlier determination [13] of the critical energy $\Delta^{*} / \mathrm{kT}$ is almost unchanged as a result of the shift in $\alpha$, leading to the value eq.14, which is $0.1 \mathrm{eV}$ smaller than previously reported [13]

$$
\Delta \mathrm{G}_{\mathrm{S}}^{\mathrm{o}}=1.75 \mathrm{eV}
$$

\section{Can Our Observations be Explained Without the Ion Evaporation Hypothesis?}

The reality of ion evaporation has been doubted by many through the long period going back to the early articles of Iribarne-Thomson. This skepticism is difficult to maintain at present, given that large ion currents coming directly from the tip of a Taylor cone held in a vacuum have been observed in a variety of experiments with formamide electrolytes [28-30]. Because the drop current was seen to decrease as the ion current increased, and because there was no time for drop evaporation, it is plain that ions are ejected directly from the meniscus tip. For liquids with electrical conductivities exceeding $1 \mathrm{~S} / \mathrm{m}$, the associated electric fields at this tip can be estimated to be of the order of $1 \mathrm{~V} / \mathrm{nm}$ [28], as predicted by the ion evaporation model. Furthermore, a purely ionic emission regime has been observed from Taylor cones of the room temperature ionic liquid 1-ethyl-3-methyl imidazolium tetrafluoroborate (EMI$\mathrm{BF}_{4}$ ). The complete absence of drops in this case shows again rather directly that the ions come from the meniscus tip, not from evaporating drops [31]. To make the point even more clear, $\mathrm{EMI}^{-\mathrm{BF}_{4}}$ is completely involatile, so that no neutral solvent evaporation could possibly fuel a Coulomb explosion. The phenomenon of ion evaporation from a charged liquid surface is therefore as indubitable as its liquid metal analog [32], in spite of the vast disparity in electrical conductivity between the liquids mentioned $(\sim 1 \mathrm{~S} / \mathrm{m})$ and liquid metals.

If the charge were to remain frozen in the drops as the solvent evaporated, the drops would then inevitably reach repeatedly the Rayleigh limit, at which they would loose charge by shedding smaller drops rather than by evaporating ions. However, since we have observed that the width of the cloud of possible charge versus diameter states encountered spans approximately one unit in the $\mathrm{z}$ axis, such hypothetical Coulomb explosions would have to always eject a single charge. Yet, Coulomb explosions from high conductivity drops tend to eject up to $50 \%$ of the drop charge (eight charges rather than one for $z=16$; [33]). Furthermore, since we know the diameter and the charge for all our residues, we can compute the ratio of their actual charge to Rayleigh's limiting charge. On these two counts the conclusion is that the charge loss observed is not due to a succession of Coulomb explosions. The fact is that the charge on our charged residues does not fit the Coulombic explosion scenario, but fits the IribarneThomson picture. While this does not prove the truth of the Iribarne-Thomson picture, it disproves the only alternative set forth so far.

\section{Conclusions}

The complex distributions of charge and mass of clusters produced from electrosprays of concentrated electrolytes have been resolved, up to $z=6$ by tandem use of a differential mobility analyzer and a mass spectrometer. The naturally formed wavy structure of the DMA-MS spectra allows extending the range of masses where $\mathrm{z}$ is known, up to $\mathrm{z}=16$ in some cases. Although the mobility analyzer used here has poor transmission efficiency, it has the advantage of being placed straightforwardly upstream of the inlet orifice of many existing mass spectrometers having an atmospheric ion source.

These experimental advantages have enabled the first simultaneous determination of mobility and mass for clusters with diameters from $1 \mathrm{~nm}$ up to nearly 9 nm. As a result:

1. We confirm the findings from prior mobility studies regarding the ability of certain concentrated salt solutions to form abundant multiply charged salt clusters, and the possibility (given sufficient resolution and sensitivity) to study such clusters by mass spectrometry.

2. Earlier expressions for the relation between size and mobility in the nanometer range have been corroborated, for the first time without ambiguity in the mass scale, though they hold only for diameters exceeding $3 \mathrm{~nm}$. At smaller sizes, the picture is complicated by what appears to be a transition from inelastic to elastic collisions that decrease the drag coefficient. This effect had been previously predicted by Tammet, though it appears at a considerably larger diameter than expected from his work (3 $\mathrm{nm}$ versus $2 \mathrm{~nm}$ ). A more complete understanding of these anomalies would enable accurate inferences of cluster density over the whole mass range from single molecules to bulk values.

3. The linearity of the relation between cluster diameter and the ion evaporation function $\mathrm{F}(\mathrm{z})$ has been verified over a range much wider than previously 
possible, confirming once more the quantitative reliability of the ion evaporation model of Iribarne and Thomson [12].

4. Improved measurements on the ion evaporation parameters $\alpha$ and $\Delta \mathrm{G}_{\mathrm{s}}^{\mathrm{o}}$ have been obtained for the tetraheptyl-ammonium ${ }^{+}$-formamide system (eqs 12 and 13).

5. A number of measurements have been made with singly and doubly charged clusters, including the first mass resolved data on their mobility up to masses approaching $9000 \mathrm{u}$. Also interesting is the discovery of the "magic cluster" $(\mathrm{AB})_{4} \mathrm{NH}_{4}^{+}$, and the observation of its anomalously high mobility in association to its symmetric structure.

6. The phenomenon of ion evaporation from solid residues reported in [13] has been confirmed, sometimes with symmetric loss of two charges.

The mechanisms investigated of initial Coulombic explosions and subsequent ion evaporation are fueled by solvent evaporation, and will surely apply to other salt-solvent combinations. The surface activity of tetraheptyl ammonium bromide has surely a large effect on the resulting level of clustering. For instance, the intense modulations at large cluster sizes observed in [15] become much weaker as the surface activity is reduced (as can be seen by studying the series of tetra-alkyl ammonium halides [34]). We do not yet know if this is due to the fact that Coulombic explosions of drops with surface active substances tend naturally to increase the salt concentration in the daughter drops [35, 36], or rather reflects the much higher stability of multiply charged clusters when their individual components are large and flexible (such as in the case discussed of arginine or tetra-peptides) than when they are small (as in $\mathrm{NaCl}$ ). That our findings are not restricted to surfaceactive salts or to formamide solutions is clearly indicated by earlier work with arginine [1] and tetrapeptides [2], both electrosprayed from aqueous solutions. In fact, the charge states reported in [2] for concentrated tetrapeptide solutions were (at fixed mass) higher than those observed here. After submission, we have become aware of a most interesting recent example showing large levels of multiply charged aggregates in the case of phospholipids [37] electrosprayed from clear aqueous suspensions with $10 \mathrm{mM}$ ammonium acetate. These mass spectra are exceptionally clean (perhaps because the analyte is neutral and the charge seems to be attributable to the small cation $\mathrm{NH}_{4}^{+}$) and resolve clearly by pure MS clusters as large as $\mathrm{D}_{75}^{+11}(\mathrm{D}=$ 1,2-dimyristoyl-sn-glycero-3-phosphocholine, has a molecular weight of $678 \mathrm{u}$ ). Testing our conclusions in such cases is just a matter of checking whether or not the corresponding cluster charges and sizes conform to the general predictions derived from the Iribarne-Thomson picture. This verification will not be possible in dilute solutions, nor on buffers unable to form large and highly charged clusters, because no solid residues would be available to bear witness of the rapid dynamics of drops.

\section{Acknowledgments}

The authors are indebted to Dr. H. M. Fales (NIH, Bethesda) for his insights on the origin of the adducts observed, to Professor Mark Johnson (Yale Chemistry) for his ideas on the probable liquid phase origin of the magic cluster $\mathrm{NH}_{4}^{+}(\mathrm{AB})_{4}$, and to Dr. Sven Ude for useful discussions and for the preparation of Figure 1. The authors gratefully acknowledge financial support from SCIEX and the United States National Science Foundation grant CTS-9871885.

\section{References}

1. (a) Meng, C. K. Multiple and fractional charging of solute molecules in electrospray ionization; Ph.D. Thesis, Yale University, 1988. (b) Meng, C. K.; Fenn, J. B. Formation of charged clusters during electrospray ionization of organic solute species. Org. Mass Spectrom. 1991, 26, 542-549.

2. Counterman, A. E.; Hildebrand, A. E.; Srebalus Barnes, C. A.; Clemmer, D. E. Formation of peptide aggregates during ESI: Size, charge, composition, and contribution to noise. J. Am. Soc. Mass Spectrom. 2001, 12, 1020-1035.

3. Anacleto, J. F.; Pleasance, S.; Boyd, R. K. Calibration of ion spray mass-spectra using cluster ions. Org. Mass Spectrom. 1992, 27, 660-666.

4. (a) Hop, C. E. C. A. Generation of high molecular weight cluster ions by electrospray ionization; implications for mass calibration. J. Mass Spectrom. 1996, 31, 1314-1316. (b) Hop, C. E. C. A.; Saulys, D. A.; Gaines, D. F. Electrospray massspectrometry of borane salts-the electrospray needle as an electrochemical-cell. J. Am. Soc. Mass Spectrom. 1995, 6, 860865. (c) Hop, C. E. C. A.; Saulys, D. A.; Gaines, D. F. Electrospray mass-spectrometry of borane salts-observation of high-molecular-weight ion clusters. Inorg. Chem. 1995, 34, 1977-1978.

5. Wang, G. D.; Cole, R. B. Solvation energy and gas-phase stability influences on alkali metal cluster ion formation in electrospray ionization mass spectrometry. Anal. Chem. 1998, 70, 873-881.

6. Winger, B. E.; Lightwahl, K. J.; Loo, R. R. O.; Udseth, H. R.; Smith, R. D. Observation and implications of high mass-tocharge ratio ions from electrospray-ionization mass-spectrometry. J. Am. Soc. Mass Spectrom. 1993, 4, 536-545.

7. Zhou, S. L.; Hamburger, M. Formation of sodium cluster ions in electrospray mass spectrometry. Rapid Commun. Mass Spectrom. 1996, 10, 797-800.

8. Zook, D. R.; Bruins, A. P. On cluster ions, ion transmission, and linear dynamic range limitations in electrospray (ionspray) mass spectrometry. Int. J. Mass Spectrom. Ion Processes 1997, 162, 129-147.

9. (a) Koch, K. J.; Gozzo, F. C.; Zhang, D. X.; Eberlin, M.N.; Cooks, R. G. Chem. Commun. 2001, 18, 1854-1855. (b) Cooks, R. G.; Zhang, D. X.; Koch, K. J.; Gozzo, F. C.; Eberlin, M. N. Chiroselective self-directed octamerization of serine: Implications for homochirogenesis. Anal. Chem. 2001, 73, 3646-3655.

10. Charles, L.; Pepin, J. C.; Gonnet, F.; Tabet, J. C. Effect of liquid phase composition on salt cluster formation in positive ion mode ESMS. Implications for clustering mechanics in electrospray. J. Am. Soc. Mass Spectrom. 2001, 12, 1067-1084.

11. Kebarle, P.; Peschke, M. On the mechanisms by which the charged droplets produced by electrospray lead to gas phase ions. Anal. Chim. Acta 2000, 406(1), 11-35. 
12. (a) Iribarne, J. V.; Thomson, B. A. On the evaporation of small ions from charged droplets. J. Chem. Phys. 1976, 64, 2287-2294. (b) Thomson, B. A.; Iribarne, J. V. Field induced ion evaporation from liquid surfaces at atmospheric pressure. J. Chem. Phys. 1979, 71, 4451-4463. (c) Thomson, B. A.; Iribarne, J. V.; Dziedzic, P. J. Liquid ion evaporation/mass spectrometry for the detection of polar labile molecules. Anal. Chem. 1982, 54, 2219-2224. (d) Thomson, B. A. Atmospheric pressure ionization and liquid chromatography mass spectrometry-together at last. J. Am. Soc. Mass Spectrom. 1998, 9, 187-193.

13. Gamero-Castaño, M.; Fernandez de la Mora, J. Kinetics of small ion evaporation from the charge and size distributions of multiply charged electrospray clusters. J. Mass Spectrom. 2000, 35, 790-803.

14. Gamero-Castaño, M.; Fernández de la Mora, J. Mechanisms of electrospray ionization of singly and multiply charged salt clusters. Anal. Chim. Acta 2000, 46, 67-91.

15. Gamero-Castaño, M.; Fernández de la Mora, J. Modulations in the abundance of salt clusters in electrosprays. Anal. Chem. 2000, 72, 1426-1429.

16. (a) Knutson, E. O.; Whitby, K. T. Aerosol classification by electric mobility: Apparatus, theory, and applications. J. Aerosol Sci. 1975, 6, 443-451. (b) Liu, B. Y. H.; Pui, D. Y. H. Submicron aerosol standard and primary, absolute calibration of condensation nuclei counter. J. Colloid Interface Sci. 1974, 47, 155-171.

17. Chernushevich, I. V.; Loboda, A. V.; Thomson, B. A. An introduction to quadrupole time-of-flight mass spectrometry. J. Mass Spectrom. 2001, 36, 849-865.

18. Fernández de la Mora, J. Free-molecule mobilities of various convex hard-bodies. J. Aerosol Sci. 2002, 33, 477-489.

19. Tammet, H. Size and mobility of nanometer particles, clusters and ions. J. Aerosol Sci. 1995, 26, 459-475.

20. (a) Fernández de la Mora, J.; de Juan, L.; Eichler, T.; Rosell, J. Differential mobility analysis of molecular ions and nanometer particles. Trends Anal. Chem. 1998, 17, 328-339. (b) Fernández de la Mora, J.; Liedtke, K.; Schmidt-Ott, A.; de Juan, L. Characterizing particles in the nanometer range by means of a mobility analyzer impactor combination. J. Aerosol Sci. 2003, 34, 79-98.

21. Friedlander, S. K. Smoke, dust, and Haze. John Wiley: New York, 1997, pp 6, 32, 36

22. (a) von Helden, G.; Wyttenbach T.; Bowers M. T. Conformation of macromolecules in the gas-phase-use of matrixassisted laser-desorption methods in ion chromatography. Science 1995, 267, 1483-1485. (b) von Helden, G.; Wyttenbach, T.; Bowers, M. T. Inclusion of a MALDI ion-source in the ion chromatography technique-conformational information on polymer and biomolecular ions. Int. J. Mass Spectrom. Ion Processes 1995, 146, 349-364. (c) Valentine, S. J.; Anderson, J. G.; Ellington, A. D.; Clemmer, D. E. J. Disulfide-intact and -reduced lysozyme in the gas phase: Conformations and pathways of folding and unfolding. Phys. Chem B 1997, 101,
3891-3900. (d) Hudgins, R. R.; Woenckhaus J.; Jarrold, M. F. High resolution ion mobility measurements for gas phase proteins: Correlation between solution phase and gas phase conformations. Int. J. Mass Spectrom. 1997, 165, 497-507. (e) Shelimov, K. B.; Clemmer, D. E.; Hudgins, R. R.; Jarrold, M. Protein structure in vacuo: Gas-phase confirmations of BPTI and cytochrome. c. J. Am. Chem. Soc. 1997, 119, 2240-2248.

23. Loscertales, I. G.; Fernández de la Mora, J. Experiments on the kinetics of field-evaporation of small ions from droplets. J. Chem. Phys. 1995, 103, 5041-5060.

24. Saucy, D.; Ude, S.; Lenggoro, W.; Fernandez de la Mora, J. Mass analysis of water soluble polymers by mobility measurement of charge-reduced electrosprays. Anal. Chem. 2004, 76, 1045-1053.

25. Ude, S. Measurement and properties of nanometer particles in the gas phase; Ph.D. Thesis, Yale University, 2004, p 144.

26. McDaniel, E. W.; Mason, E. A. The mobility of ions in gases; Wiley: New York, 1973, p 46.

27. Labowsky, M.; Fenn, J. B.; Fernandez de la Mora, J. A continuum model for ion evaporation from a drop: Effect of curvature and charge on ion solvation energy. Anal. Chim. Acta 2000, 406, 105-118.

28. Gamero-Castaño, M.; Fernández de la Mora, J. Direct measurement of ion evaporation kinetics from electrified liquid surfaces. J. Chem. Phys. 2000, 113, 815-832.

29. Gamero-Castaño, M; Hruby, V. Electrospray as a source of nanoparticles for efficient colloid thrusters. J. Propulsion Power 2001, 17, 977-987.

30. Bocanegra, R.; Fernandez de la Mora, J.; Gamero, M. Ammonium electrolytes quench ion evaporation in colloidal propulsion. J. Propulsion Power 2004, 20(4), 728-735.

31. Romero-Sanz, I.; Fernandez de la Mora, J. Spatial structure and energy distribution of electrosprays of ionic liquids in vacuo. J. Appl. Phys. 2004, 95(4), 2123-2129.

32. Prewett, P. D.; Mair, G. L. R. Focused ion beams from liquid metal ion sources; Wiley: New York, 1991.

33. Richardson, C. B.; Pigg, A. L.; Hightower, R. L. Proc. R. Soc. London A 1989, 422, 319.

34. Ku, B. K., Fernandez de la Mora, J. Ion evaporation kinetics of tetra-alkylammonium salts in formamide solution. J. Phys. Chem., submitted.

35. Tang, L.; Kebarle, P. Dependence of ion intensity in electrospray mass-spectrometry on the concentration of the analytes in the electrosprayed solution. Anal. Chem. 1993, $65,3654-3668$. (section $g$, page 3666)

36. Verkerk, U. H.; Peschke, M.; Kebarle, P. Effect of buffer cations and of $\mathrm{H}_{3} \mathrm{O}^{+}$on the charge states of native proteins. Significance to determinations of stability constants of protein complexes. J. Mass Spectrom. 2003, 38, 618-631.

37. Hanson, C. L.; Ilag, L. L.; Malo, J.; Hatters, D. M.; Howlett, G. J.; Robinson, C. V. Phospholipid complexation and association with apolipoprotein C-II: Insights from mass spectrometry. Biophys. J. 2003, 85, 3802-3812. 